\title{
Consideration of Hysteresis, Saturation, Fringing and Leakage Fluxes in the Frequency-Dependent Analytical Model of Nonlaminated Cylindrical Actuators
}

\author{
Robert Seifert ${ }^{1 *}$, Johannes Porstmann ${ }^{2 *}$ and Wilfried Hofmann ${ }^{1}$, Senior Member, IEEE \\ ${ }^{1}$ Chair of Electrical Machines and Drives, Technische Universität Dresden, 01069 Dresden, Germany \\ ${ }^{2}$ Fraunhofer Institute for Transportation and Infrastructure Systems IVI, 01069 Dresden, Germany
}

\begin{abstract}
Previous works have demonstrated that analytical high-fidelity models of nonlaminated actuators and magnetic thrust bearings cannot just describe the magnetic skin effect inside the solid core, but also be applied directly within the control circuit. By an appropriate rational approximation a digital implementation on a microcontroller becomes possible. However, these approximated models neither considered hysteresis and saturation nor frequency-dependent fringing and leakage fluxes. This article elaborates whether or not these nonlinearities can and should be included in real-time control systems. We present an improved process to map an analytical hysteresis model to a limited measured dataset and discuss the impact of the nonlinear magnetization curve. It leads to a novel fractional-order all-pass filter, modeling the frequency-dependent hysteresis angle for a single load point. Its rational filter form is suitable for implementation in MATLAB/SIMULINK as well as real-time applications. Leakage and fringing fluxes, on the other hand, can be considered with relatively low effort within the original analytical models. The underlying reluctance network is determined by a FE-analysis as well as analytically and reduced to a highly simplified form. Depending on whether the total flux or the force-dependent flux is of interest, the model order may increase significantly and constant correction factors are preferable.
\end{abstract}

\section{Index Terms-Actuators, Analytical Modeling, Hysteresis, Leakage Fluxes, Magnetic Bearings}

\section{INTRODUCTION}

In recent years magnetic bearings have seen an increase in their industrial applications due to a reduction in costs, of not only, the required power electronics and controllers but also in maintenance and operations. This has prompted traditional manufacturers of mechanical bearings and other mass products, such as compressors, pumps and turbines to move further into this market. As a consequence, there emerges a desire for high-stiffness magnetic bearings with a disturbance suppression $\Delta K_{\mathrm{DS}}>120 \mathrm{~dB}$, which will match the stiffness of their mechanical counterparts $\left(\Delta K_{\mathrm{DS}} \approx 160 \mathrm{~dB}\right)$ to some degree. This is especially challenging for magnetic thrust bearings as well as other solid-core actuators, as eddy current flow inside the iron core and the resultant magnetic skin effect [1] is usually not avoidable. The three-dimensional flux propagation renders a laminated core, as common in radial bearings, ineffective. An axially slit core [2] as well as the use of soft magnetic composites [3] can be considered as too expensive and mechanically weak for most applications.

The magnetic skin effect causes a significant lag between the force-generating magnetic flux and the measurable coil current, which results into a substantial damping of the actuator force in the usually current controlled systems [4]. However, alternative control topologies, like $\mathrm{H}_{\infty}$-control [5], state-control with Kalman-filters [6] or direct voltage control [7] as well as hybrid forms [8], [9] could not assert themselves. The reasons for this are of a mainly practical nature. Unlike its mentioned alternatives, the common cascaded position control with subordinated current control [10], is the only approach

\footnotetext{
${ }_{*}^{1}$ Corresponding author: R. Seifert (email: robert.seifert@tu-dresden.de).

${ }^{*}$ R. Seifert and J. Porstmann contributed equally to this article.

Digital Object Identifier: 10.36227/techrxiv.14473641

This manuscript is not peer-reviewed and may be subject to changes.
}

to maintain the underlying physics within the control circuit. It relies on typical parameters like the stiffness $k$ and the damping $d$, which are equal to ball, oil and air bearings, and therefore keep the initial start-up of the magnetic bearing simple and comprehensible. For this reasons we presented an alternative control approach in our previous work [1], maintaining the cascaded structure and its practical advantages. Instead of the measurable coil current, we control the actual force-generating flux density as shown in Fig. 1. In the feedback branch we introduced a fractional-order flux estimator, which is able - in its rational form - to determine the air gap flux density from the measurable coil current in real-time. By use of the diffusion equation, the flux estimator considers the magnetic skin effect caused by eddy currents, but neglects other nonlinearities, such as hysteresis, core saturation and fringing as well as leakage fluxes. This article aims to include the missing nonlinearities into the high-fidelity analytical model behind the flux density estimator. While it is straightforward in case of the fringing and leakage fluxes, by means of a simplified reluctance network, it remains challenging for hysteresis and the nonlinear magnetization curve. We will discuss why their consideration is not entirely possible for every case.

This paper is organized as follows. Section II gives a brief introduction into the existing eddy-current model, on which the flux density estimator is based on. For a deeper understanding of the physics and the mathematical modeling based on fractionalorder systems, we like to refer to our previous work [1]. In section III we will first delve briefly into the historic modeling of hysteresis before focusing on the identification of the loaddependent hysteresis angle $\vartheta_{\text {hyst }}$ in section IV. We start by modeling the major hysteresis loop, which is then used to find the so-called first order reversal curves and minor loops to obtain a load-dependent hysteresis description. A frequency- 


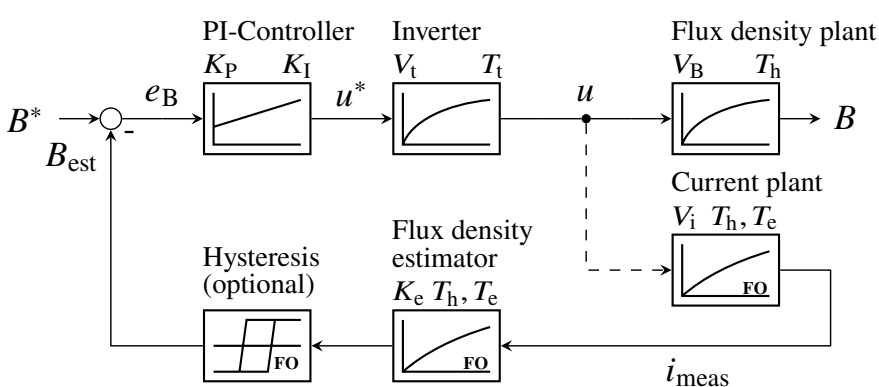

Fig. 1. Closed-loop flux density control with fractional-order flux estimator based on the measured coil current $i_{\text {meas }}$ and optional fractional-order all-pass hysteresis filter $-K_{i}$ : controller and estimator gains, $V_{i}$ : plant gains, $T_{i}$ : plant delays, FO: fractional-order systems

dependency is achieved in section $\mathrm{V}$ by adapting the eddycurrent model by use of the hysteresis angle $\vartheta_{\text {hyst }}$, which allows us to identify a fractional-order all-pass hysteresis filter, which may be used within actuator control. We conclude the first part of this article with a discussion about the applicability of the hysteresis' load-dependency and the core saturation.

The second part of the article is dedicated to the consideration of fringing and leakage fluxes. We use a FE-analysis to identify all flux paths and form a reluctance network for the static case in section VI. The inverted model can then be used in section VII to calculate all frequency-dependent fringing/leakage fluxes for a given magnetomotive force. We conclude with a proposal for a corrected analytical model, to be used with the real-time flux density control.

\section{EDDY CURRENT EFFECTS AND THEIR MODELING}

Eddy currents in nonlaminated cylindrical actuators have already been discussed thoroughly in the literature in recent years. The foundations of any high-fidelity model in the frequency-domain were laid down by Stoll [11], further refined by Feeley [12] to establish the so-called "eddy-inductance", which we used for our flux density estimator. Cylindrical actuators and magnetic bearings were first considered by [13] and [14], respectively, leading to the almost complete model by Zhu et al. [4]. In our previous work [1] we presented a full historical review of eddy current models, a study on their physical impact as well as final model refinements. Due to this extensive groundwork we will introduce only the most essential equations here.

In a first step, we divide the actuator geometry (Fig. 2) into core parts, which are only permeated by a one-dimensional magnetic flux $\Phi_{i}(\mathrm{j} \omega)$. This we calculate by solving the electromagnetic diffusion equation in its complex form

$$
\operatorname{curl}(\operatorname{curl} \underline{\vec{B}})=-\mathrm{j} \omega \kappa \mu \underline{\vec{B}}=-\underline{\alpha}^{2} \underline{\vec{B}}
$$

for every core element, where $\underline{\alpha}=\sqrt{\mathrm{j} \omega \kappa \mu}$ denotes the complex wave propagation constant with the constant permeability $\mu=\mu_{0} \mu_{\mathrm{r}}$ and electrical conductivity $\kappa$ (for assumed isotropic and homogeneous materials). By help of the magnetomotive force (mmf) $\underline{\Theta}(\mathrm{j} \omega)$, we obtain the overall effective reluctance

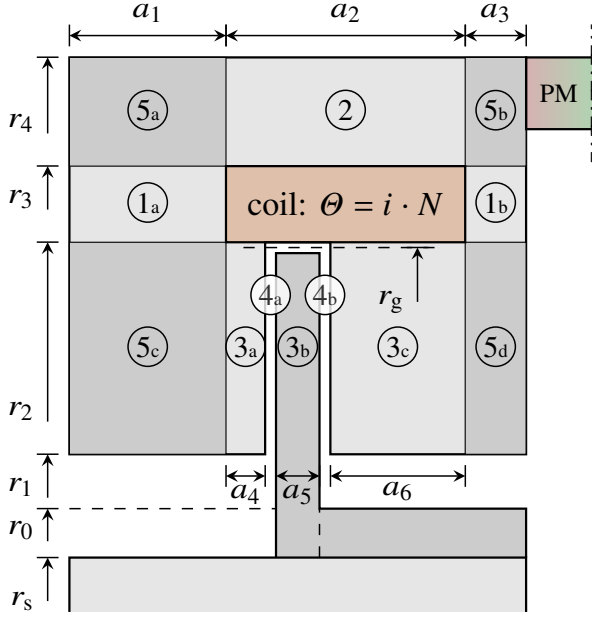

\begin{tabular}{cccc}
\hline & $\frac{\lambda_{i}}{\mathrm{~mm}}$ & & $\frac{r_{i}}{\mathrm{~mm}}$ \\
\hline$a_{1}$ & 14.4 & $r_{0}$ & 17.5 \\
$a_{2}$ & 22.0 & $r_{1}$ & 22.5 \\
$a_{3}$ & 5.6 & $r_{2}$ & 42.0 \\
$a_{4}$ & 3.6 & $r_{3}$ & 52.0 \\
$a_{5}$ & 5.0 & $r_{4}$ & 59.0 \\
$a_{6}$ & 12.4 & $r_{\mathrm{g}}$ & 41.8 \\
$g$ & 0.5 & $r_{\mathrm{s}}$ & 13.0 \\
\hline
\end{tabular}

(1) $\mathcal{R}_{\mathrm{r}}$ (2) $\mathcal{R}_{\mathrm{ao}}$

(3) $\mathcal{R}_{\mathrm{ai}}$ (4) $\mathcal{R}_{\mathrm{gi}}$

(5) $\mathcal{R}_{\mathrm{c}}$

Fig. 2. Magnetic circuit of thrust bearing divided into five classes of effective reluctances - core material: $15 \mathrm{NiCr} 13 / 1.5752$ with $\mu_{\mathrm{r}}=880, \kappa=5 \mathrm{MS} \mathrm{m}^{-1}$ $-N$ : coil turns, $i$ : coil current, adapted from [1]

$\underline{\mathcal{R}}_{\text {eff }}(\mathrm{j} \omega)$ as the sum of the part reluctances $\underline{\mathcal{R}}_{i}(\mathrm{j} \omega)$ of every core element:

$$
\underline{\mathcal{R}}_{\mathrm{eff}}(\mathrm{j} \omega)=\frac{N^{2}}{\left.\underline{L}_{\mathrm{eff}} \mathrm{j} \omega\right)}=\sum_{i} \frac{\underline{\Theta}(\mathrm{j} \omega)}{\underline{\Phi}_{i}(\mathrm{j} \omega)}=\sum_{i} \underline{\mathcal{R}}_{i}(\mathrm{j} \omega),
$$

which translates into the effective inductance $\underline{L}_{\mathrm{eff}}(\mathrm{j} \omega)$ and the definition of the desired flux density estimator

$$
\underline{G}_{\mathrm{FE}}(\mathrm{j} \omega)=\frac{\underline{B}_{\mathrm{est}}(\mathrm{j} \omega)}{\underline{i}_{\text {meas }}(\mathrm{j} \omega)}=\frac{L_{\mathrm{h}}}{N A_{\mathrm{g}}} \frac{R_{\mathrm{Cu}}+s \underline{L}_{\mathrm{eff}}(\mathrm{j} \omega)}{R_{\mathrm{Cu}}+s L_{\mathrm{h}}},
$$

with the air gap area $A_{\mathrm{g}}$ and the number of coil turns $N$. The main inductance $L_{\mathrm{h}}=L_{\text {eff }}(\omega=0)$ corresponds to the forcegenerating component of current and flux, which is independent from the eddy currents. As an example we regard an toroidal core as studied in [15] with the core radius $r_{\mathrm{c}}$ and the flux path circumference $\lambda_{\mathrm{c}}$, whose effective reluctance $\underline{\mathcal{R}}_{\text {eff }}$ can be described with the transcendent function

$$
\underline{\mathcal{R}}_{\mathrm{eff}}(\mathrm{j} \omega)=\frac{\underline{\alpha}}{2 \pi \mu} \frac{\lambda_{\mathrm{c}}}{r_{\mathrm{c}}} \frac{\mathrm{I}_{0}\left(\underline{\alpha} r_{\mathrm{c}}\right)}{\mathrm{I}_{1}\left(\underline{\alpha} r_{\mathrm{c}}\right)}=\frac{\sqrt{\mathrm{j} \omega \kappa}}{2 \pi \sqrt{\mu}} \frac{\lambda_{\mathrm{c}}}{r_{\mathrm{c}}} \frac{\mathrm{I}_{0}\left(\sqrt{\mathrm{j} \omega \kappa \mu} r_{\mathrm{c}}\right)}{\mathrm{I}_{1}\left(\sqrt{\mathrm{j} \omega \kappa \mu} r_{\mathrm{c}}\right)} .
$$

The same formula also applies approximately for the core elements of class 3 in Fig. 2 with $r_{\mathrm{c}}=\left(r_{2}^{2}-r_{1}^{2}\right) / r_{2}$ as defined in [1]. In the following section $\mathrm{V}$, we will use this example to demonstrate the expansion of any transcendental solution with the hysteresis angle $\vartheta_{\text {hyst }}$ to model the frequency-dependent impact of the hysteresis.

\section{REVIEW OF HYSTERESIS MODELS}

When discussing hysteresis models, we have to distinguish between two groups. First, the static model of a single or a set of hysteresis loops, which we use to identify characteristic parameters to describe hysteresis-related material properties. And secondly, in section V, we adopt these parameters to create a frequency- or load-dependent description for the actuator control or transient models.

The hysteresis in electromagnetic materials has been a subject of scientific study since to the beginning of the 20th century. The work of Preisach [16] showed that there exists a phase 
TABLE I

OVERVIEW OF THE MAGNETIC MATERIALS EXAMINED IN THE REFERENCED LITERATURE

\begin{tabular}{|c|c|c|c|c|c|c|}
\hline Reference & Material & $\frac{H_{\max }}{\mathrm{A} / \mathrm{m}}$ & $\frac{B_{\max }}{\mathrm{T}}$ & $\mu_{\mathrm{rBr}}$ & $\mu_{\mathrm{rHc}}$ & $\mu_{\text {r tip }}$ \\
\hline \multicolumn{7}{|l|}{ LAMINATED CORES } \\
\hline Jiles $[24]$ & Permalloy 80 & 10 & 1.05 & 27500 & $1 \cdot 10^{6}$ & 1250 \\
\hline Cardelli [21] & & 250 & 0.43 & 5629 & 9229 & 1359 \\
\hline Cardelli [22] & MnZn Ferrite & 575 & 0.50 & 1486 & 1649 & 725 \\
\hline Cardelli [25] & & 100 & 0.30 & 2900 & 12391 & 2476 \\
\hline \multirow{3}{*}{ Ribbenfjärd [26] } & $\mathrm{M}^{\mathrm{a}}$ & 1350 & 1.43 & 8240 & 13685 & 875 \\
\hline & M600-50A & 1250 & 1.33 & 2689 & 9632 & 864 \\
\hline & $\mathrm{ZDKH}^{\mathrm{a}}$ & 1300 & 1.45 & 14129 & 30384 & 1158 \\
\hline \multirow{2}{*}{ Milovanovic [27] } & electrical steel ${ }^{\mathrm{a}}$ & 300 & 1.60 & 8127 & 79350 & 4363 \\
\hline & MnZn Ferrite & 210 & 0.24 & 962 & 8158 & 961 \\
\hline \multicolumn{7}{|l|}{ SOLID CORES } \\
\hline Rodriguez [28] & low-carbon steel & 2000 & 1.40 & 322 & 356 & 151 \\
\hline this article & $15 \mathrm{NiCr} 13$ & 28000 & 2.06 & 133 & 8620 & 59 \\
\hline
\end{tabular}

${ }^{\mathrm{a}}$ Grain oriented electrical steel, otherwise nonoriented or unknown

lag $\vartheta_{\text {hyst }}$ between an applied external magnetic field $H_{\text {ext }}$ and the resulting magnetic induction $B$ within an electromagnetic material. Preisachs modeling of his observation has since been known as the Preisach-model. With the advent of magnetic storage devices in the 1980s, a further understanding of hysteresis modeling was necessary. Krasnosel'skii et al. [17] showed the general phenomenological nature of the Preisach-model, which in turn enabled Mayergoyz et al. [18] to formulate its memory and congruence properties. Furthermore, Mayergoyz noted that the determination of the density function for the Preisach-model needs to be based on measured first order reversal curves (FORCs).

Since Mayergoyz's work, several augmentations to the basic Preisach-model have been attempted to better reflect observations. In order to describe the dynamic behavior of hysteric loops, Bertotti [19] expanded the model to reflect the widening of hysteric loops due to an increasing frequency. Della Torre and Vajda [20] introduced the complete-moving hysteresis model to include the reversible and irreversible aspects of magnetic material behavior. After the introduction of the modified scalar Preisach model in [21], Cardelli et al. established the concept of the equivalent ellipse in [22] to easily determine the phase lag of a specific material as we will further discuss in section V. Even though the study of the Preisach-model has a long history, its greatest limitation for a broader application is the inherent difficulty in determining the necessary density function ideally based on measured FORCs. Novak [23] noted, that these are only measurable with highly precise instruments and a considerable amount of data post processing.

Alternative models for hysteresis modeling have also been introduced, for one, from Jiles and Atherton [29]. The JilesAtherton model utilizes a physical instead of a phenomenological approach and considers the domain wall motion under magnetization to describe the hysteresis behavior. While an expansion of the model exists to analyze the dynamic behavior, it is emphasized that the model is best suited for thin laminated sheets [24]. Another attempt to describe frequency dependent hysteresis followed by Ribbenfjärd and Engdahl [26] who used the work of Bertotti [30] to introduce a Cauer network. Again, special emphasis is placed on using the model for thin sheets exclusively. Nevertheless, Herzog et al. [31] used Ribbenfärd's approach to examine a solid core axial magnetic bearing. Even though both Ribbenfjärd and Herzog were able to achieve a good parity between model and measurements, both pointed out that the model parameter identification process needs to be improved.

To circumvent the inherent identification difficulties of the model parameters, Faiz and Saffari [32] used a neural network approach to construct load-dependent hysteresis loops. Using their method, Milovanovic and Koprivica [27] proposed an arc tangent based model, which we will focus on in this paper. Within the here presented literature, a variety of different materials were examined, which are listed in Table I. They show a wide range of applied maximum field strengths $H_{\max }$ and measured flux densities $B_{\max }$ leading to the highly varying absolute permeability $\mu_{\mathrm{rtip}}=B_{\max } /\left(\mu_{0} H_{\mathrm{max}}\right)$, and differential permeabilities $\mu_{\mathrm{r} B r}$ and $\mu_{\mathrm{r}} \mathrm{Hc}$. It becomes apparent, that most of the previous studies examined electrical steel sheets, so very few experiences with the hysteresis in nonlaminated cores were made.

\section{Static Hysteresis Model}

The static hysteresis model we apply in this article is based on the work of Milovanovic et al. [27] for two reasons. First, due to limited technical possibilities, we can only measure the major hysteresis loop and the initial magnetization curve of our applied core material $15 \mathrm{NiCr} 13$, which in consequence forbids the Preisach-model and its variations. Second, the desired hysteresis angle $\vartheta_{\text {hyst }}$ is mainly determined by the area enclosed by the hysteresis loop, so its accurate representation is more important than the simplicity or applicability of the static model. We do not exclude the possibility, that other approaches may lead to equally satisfying results, but from the models we studied and mentioned in section III, Milovanovic's model is by far the most suitable for our case.

\section{A. Major Loop}

Given a data set consisting of a semi-static hysteresis loop (henceforth defined as the major loop $B_{\text {ma }}$ ) and the initial magnetization curve, it is of fundamental importance for a frequency analysis to be able to determine the resulting loop for any applied load $\hat{H}_{\text {ap }}$. Presuming that a major loop reflects the behavior of a fully saturated material $\left(\hat{H}_{\max }=\hat{H}_{\text {sat }}\right)$, any resulting closed loop given an applied field $\hat{H}_{\text {ap }}<\hat{H}_{\text {sat }}$ will be defined as a minor loop $B_{\mathrm{mi}}(H)$. The major loop $B_{\mathrm{ma}}(H)$ and the initial magnetization curve $B_{\mathrm{vir}}(H)$ are shown in Fig. 3 . The identification process can be simplified by mirroring the descending branch at the origin to match the ascending branch and average both curves to obtain a symmetrized major loop. 

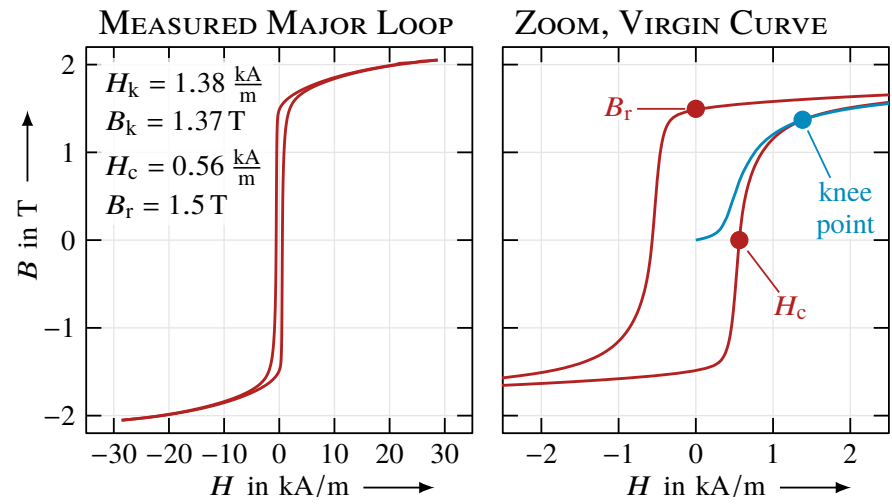

Fig. 3. Major loop and initial magnetization curve of $15 \mathrm{NiCr} 13$, measurements carried out by Sekels GmbH with REMAGRAPH ${ }^{\circledR}$ by Dr. Steingroever GmbH Knee point definition by IEC 60050-321:1986

The model proposed by Milovanovic et al. [27] divides the major loop into an ascending $B_{\mathrm{A}}(H)$ and descending branch $B_{\mathrm{D}}(H)$ so that it can be described by

$$
\begin{aligned}
& B_{\mathrm{A}}(H)=a \cdot \arctan \left(b \cdot\left(H-H_{\mathrm{c}}\right)\right)+c \cdot H \\
& B_{\mathrm{D}}(H)=a \cdot \arctan \left(b \cdot\left(H+H_{\mathrm{c}}\right)\right)+c \cdot H
\end{aligned}
$$

The three coefficients $a, b$ and $c$ represent parameters based on material properties (Fig. 4). The parameter $a$ is the saturated flux density $B_{\text {sat }}$ scaled to the maximum of the arctan-function and the parameter $b$ adapts the slope $a \cdot b$ of the function for $B<B_{\mathrm{r}}$, such that

$$
a=B_{\mathrm{sat}}^{\prime}=\frac{2}{\pi} \cdot B_{\mathrm{sat}} \quad \text { and } \quad b=\frac{\tan \left(B_{\mathrm{r}} / B_{\mathrm{sat}}^{\prime}\right)}{H_{\mathrm{c}}} .
$$

The parameter $c$ models the slope for $B>B_{\mathrm{r}}$

$$
c=\frac{B_{\mathrm{sat}}^{\prime}}{H_{\mathrm{sat}}} \cdot\left(\frac{\pi}{2}-1\right) \cdot \arctan \left(b \cdot\left(H_{\mathrm{sat}}+H_{\mathrm{c}}\right)\right) .
$$

By inserting (7) and (8) into (5) and (6), it is possible to represent an entire major loop. However, depending on the material this may not accurately represent the enclosed area of the given major loop. Since it can not be assumed that a major loop shows point symmetry in respect to $\pm H_{\mathrm{c}}$, [27] demonstrated that further separating the individual branches $B_{\mathrm{A}}$ and $B_{\mathrm{D}}$ allows for a far more accurate model. A branch is divided into its positive and negative flux density values such that the complete major loop can be expressed as

$$
B_{\mathrm{A}}(H)=\left\{\begin{array}{l}
a_{\mathrm{n}} \arctan \left(b_{\mathrm{n}}\left(H-H_{\mathrm{c}}\right)\right)+c_{\mathrm{n}} H \text { for } H \leq H_{\mathrm{c}} \\
a_{\mathrm{p}} \arctan \left(b_{\mathrm{p}}\left(H-H_{\mathrm{c}}\right)\right)+c_{\mathrm{p}} H \text { for } H_{\mathrm{c}}<H,
\end{array}\right.
$$

for the ascending branch and

$$
B_{\mathrm{D}}(H)=\left\{\begin{array}{l}
a_{\mathrm{p}} \arctan \left(b_{\mathrm{p}}\left(H+H_{\mathrm{c}}\right)\right)+c_{\mathrm{p}} H \text { for } H \leq-H_{\mathrm{c}} \\
a_{\mathrm{n}} \arctan \left(b_{\mathrm{n}}\left(H+H_{\mathrm{c}}\right)\right)+c_{\mathrm{n}} H \text { for }-H_{\mathrm{c}}<H
\end{array}\right.
$$

for the descending branch. Utilizing a nonlinear least square regression, with equations (7) and (8) as the appropriate starting parameters, it is possible to determine the values for the parameters $a_{\mathrm{n}, \mathrm{p}}, b_{\mathrm{n}, \mathrm{p}}$ and $c_{\mathrm{n}, \mathrm{p}}$, which do not differ for the ascending and descending case, due to the previous
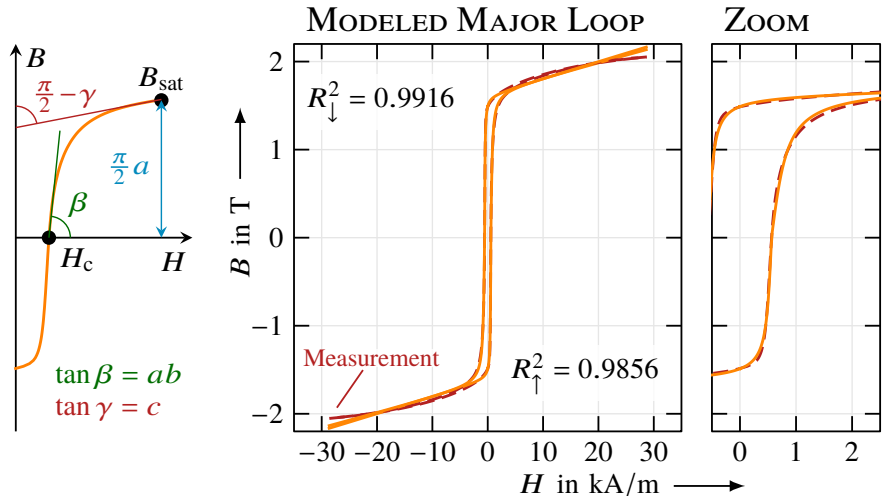

Fig. 4. Parameter definition of arctan-model leading to modeled ascending $B_{\mathrm{A}}$ and descending $B_{\mathrm{D}}$ branch in comparison with measured major loop for $15 \mathrm{NiCr} 13$ steel $-R^{2}$ : coefficient of determination

symmetrization of the major loop. For the examined $15 \mathrm{NiCr} 13$ steel it leads to the fit as illustrated in Fig. 4, whose enclosed area shows an relative error of only $1.38 \%$ compared to the measured loop.

\section{B. First Order Reversal Curves}

Since the major loop describes the hysteresis of a material which undergoes negative and positive saturation, all information pertaining to the applied field strengths less than the saturation field strength is essentially lost. However, it is possible to model the minor loops with the aid of so-called first order reversal curves (FORCs). These curves are defined as follows: if a material is subject to an applied field which decreases from its saturation value, but again increase before it reaches its negative saturation value and continues to increase back to its positive saturation value, the resulting magnetization curve is an ascending FORC [30] and henceforth defined as

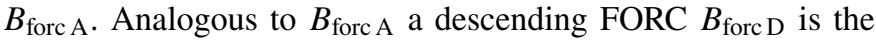
result of an applied field first increasing and subsequently decreasing. Regardless of which curve is examined, the point at which the applied field changes its polarity will be defined as the reversal point $H_{\mathrm{rev}}$. This point can be viewed as the specific load point that is proportional to the applied current $\hat{I}$, the number of coil turns $N$ and the length of the core $\lambda_{\mathrm{c}}$ with $H_{\text {rev }}=\hat{I} \cdot N / \lambda_{\mathrm{c}}$. Further following [27] who proposed for an ascending FORC:

$$
B_{\text {forc A }}(H)=B_{\mathrm{A}}(H)+s_{\mathrm{A}}(H) \text { for }|H| \leq\left|H_{\text {rev }}\right| \text {, }
$$

we can construct the ascending branch of the FORC by using the shifting factor $s_{\mathrm{A}}(H)$, which is defined as

$$
s_{\mathrm{A}}(H)=\overbrace{\left(\left|B_{\text {rev A }}\right|-\left|B_{\text {rev D }}\right|\right)}^{s_{\text {max }}}\left(\frac{B_{\mathrm{A}}(H)-B_{\mathrm{s}}}{B_{\text {rev A }}-B_{\mathrm{s}}}\right)^{1+\beta B_{\mathrm{D}}(H)} .
$$

The terms $B_{\text {rev A|D }}$ express the corresponding flux densities at the reversal point. The FORC parameter $\beta$ is a degree of freedom, which needs to be determined. While [27] used a graphical approach, we will calculate $\beta$ analytically by the following steps and the visual guidance in Fig. 5a. Firstly, 


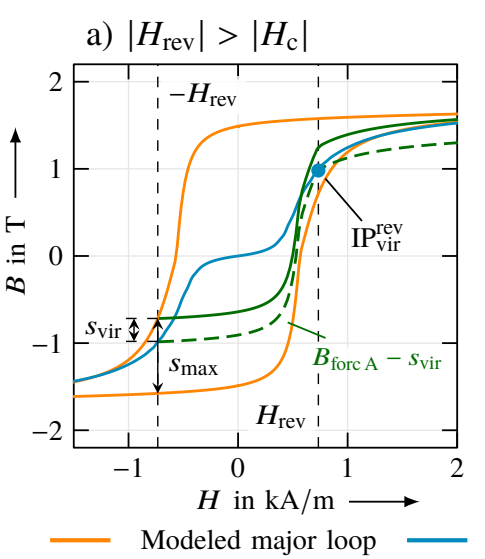

b) $\left|H_{\text {rev }}\right| \leq\left|H_{\mathrm{c}}\right|$

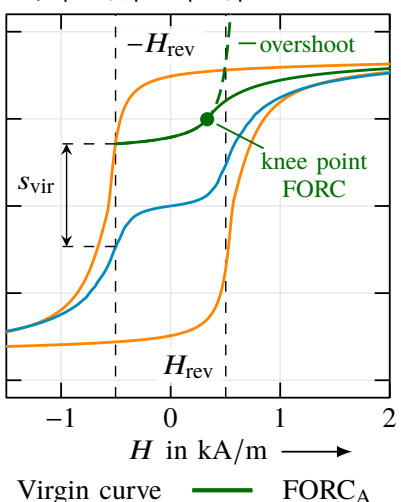

Fig. 5. Visualization for the modeling of the first order reversal curves for a) $\left|H_{\text {rev }}\right|>\left|H_{\mathrm{c}}\right|$ and b) $\left|H_{\text {rev }}\right| \leq\left|H_{\mathrm{c}}\right|$

$B_{\text {forc A }}(H)$ is vertically shifted by $s_{\text {vir }}$ to the equivalent reversal point on the inverted virgin curve $B_{\text {vir }}^{\prime}$ such that

$$
S_{\mathrm{vir}}=\underbrace{B_{\text {forc A }}\left( \pm H_{\mathrm{rev}}\right)}_{B_{\mathrm{D}}\left( \pm H_{\mathrm{rev}}\right)}-B_{\mathrm{vir}}^{\prime}\left( \pm H_{\mathrm{rev}}\right)
$$

which holds true for the positive as well as negative reversal point $\pm H_{\text {rev }}$. By inserting (13) into (12) we can finally solve for $\beta$ :

$\beta=\frac{1}{B_{\mathrm{D}}\left(H_{\mathrm{rev}}\right)}\left[\frac{\ln \left(\frac{B_{\mathrm{vir}}\left(H_{\mathrm{rev}}\right)-s_{\mathrm{vir}}-B_{\mathrm{A}}\left(H_{\mathrm{rev}}\right)}{s_{\mathrm{max}}}\right)}{\ln \left(\frac{B_{\mathrm{A}}\left(H_{\mathrm{rev}}\right)-B_{\mathrm{s}}}{B_{\mathrm{A}}\left(-H_{\mathrm{rev}}\right)-B_{\mathrm{s}}}\right)}-1\right]$.

Depending on the material and the reversal point, the modeled FORC can exceed the major loop for $H_{\text {rev }}>H_{\mathrm{c}}$. While Milovanovic solved this dilemma with the introduction of a further parameter, we propose an equally effective horizontal shift using known parameters instead, such that

$$
\Delta H_{\text {rev }}=H_{\text {rev }}-\frac{1}{b_{\mathrm{p}}} \cdot \tan \left(\frac{B_{\text {forc A }}\left(H_{\text {rev }}\right)-c_{\mathrm{p}} \cdot H_{\text {rev }}}{a_{\mathrm{p}}}\right) .
$$

However, in case the reversal points $H_{\text {rev }}$ are smaller than the major loop coercivity $H_{\mathrm{c}}$, the overshoot correction in (15) becomes ineffective as illustrated in Fig. 5b. To resolve that, we propose a switchover point $H_{\mathrm{so}}$ smaller than $H_{\text {rev }}$ and introduce the FORC knee point $H_{\text {forc k }}$. The general knee point is defined in IEC 60050-321:1986 321-02-34 for current transformers as the voltage (or field strength), whose further increase by $10 \%$, would cause an increase of the exciting current (or induction) by $50 \%$. We usually use this definition to demarcate the assumed linear part from the saturated part of the virgin curve (cf. Fig. 3). In case of the FORCs, we propose to identify the knee point field strength $H_{\text {forc }}$, whose decrease by the ratio $H_{\text {rev }} / H_{\mathrm{c}}$ would cause a decrease of the flux density by the ratio $\left(B_{\mathrm{r}}-B_{\mathrm{D}}\left(-H_{\mathrm{rev}}\right)\right) / B_{\mathrm{r}}$, so that the relationship

$$
H_{\text {forc k }}\left(\left[1-\frac{B_{\mathrm{D}}\left(-H_{\text {rev }}\right)}{B_{\mathrm{r}}}\right] B_{\text {forc k }}\right)=\frac{H_{\text {rev }}}{H_{\mathrm{c}}} \cdot H_{\text {forc k }}\left(B_{\text {forc k }}\right)
$$

TABLE II

Necessary EQUations for MOdeling First ORder Reversal CuRves baSed ON MODELED ASCENDING AND DESCENDING MAJOR LOOP CURVES

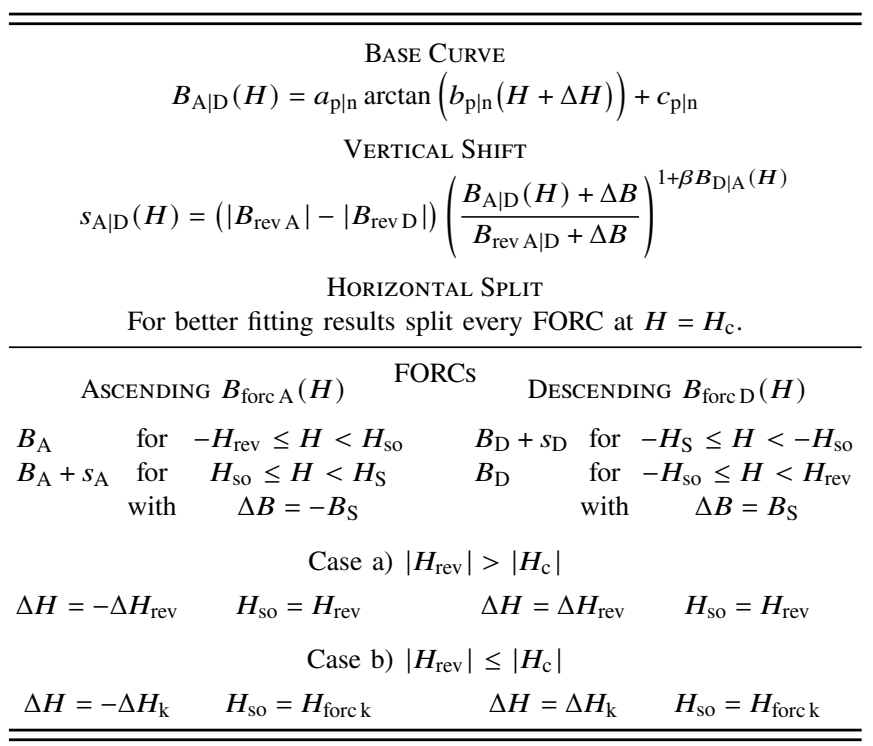

holds true and the knee point $H_{\text {forc k }}, B_{\text {forck }}$ can be determined numerically. The horizontal shift parameter in (15) is adapted accordingly by substitution of $H_{\text {rev }}$ with $H_{\text {forc k }}$ :

$$
\Delta H_{\mathrm{k}}=H_{\text {forc k }}-\frac{1}{b_{\mathrm{p}}} \cdot \tan \left(\frac{B_{\text {forc k }}-c_{\mathrm{p}} \cdot H_{\text {forc k }}}{a_{\mathrm{p}}}\right),
$$

for the case $\left|H_{\text {rev }}\right| \leq\left|H_{\mathrm{c}}\right|$. The FORC for the ascending case will therefore have the form

$$
B_{\text {forc A }}(H)=a_{\mathrm{p}} \cdot \arctan \left(b_{\mathrm{p}} \cdot(H-\Delta H)\right)+c_{\mathrm{p}} \cdot H \text {. }
$$

Since modeling the descending FORC is analogous to the ascending, it will not be further derived in the context of this article. A summary of the necessary equations to model both $B_{\text {forc A }}$ and $B_{\text {forc D }}$ is listed in Table II. All scalar parameters such as $\beta, s_{\mathrm{vir}}$ and $s_{\max }$ as well as $\Delta H_{\mathrm{rev}}$ and $\Delta H_{\mathrm{k}}$ are identical for both cases, due to symmetry. With the herein presented procedure it is now possible to model every FORC $B_{\text {forc } \mathrm{A} \mid \mathrm{D}}$ for any reversal point $H_{\text {rev }}$ and therefore any load $I$.

\section{Minor Loops}

With the ascending and descending FORC model it is now possible to determine the hysteresis of a material for any load point. In order to construct a minor loop, Faiz and Saffari [32] showed that this is possible by vertically shifting an FORC to the point where it intersects its opposite FORC. Building on the congruence property proposed by Mayergoyz [18] a shift back of the resulting closed loop, centered around the origin, gives a minor loop. The procedure is illustrated in Fig. 6.

In a first step, we shift $B_{\text {forc D }}$ to the point that it intersects $B_{\text {forc A }}$ using the factor $s_{\mathrm{mi}}$, which is defined as

$$
s_{\mathrm{mi}}=B_{\text {forc A }}\left(H_{\text {rev }}\right)-B_{\text {forc D }}\left(H_{\text {rev }}\right),
$$

to obtain a displaced minor loop, which can be centered by the second shift factor

$$
s_{\mathrm{sym}}=\frac{B_{\text {forc A }}\left(H_{\mathrm{rev}}\right)+B_{\text {forc A }}\left(-H_{\mathrm{rev}}\right)}{2}=\frac{s_{\mathrm{mi}}}{2} .
$$



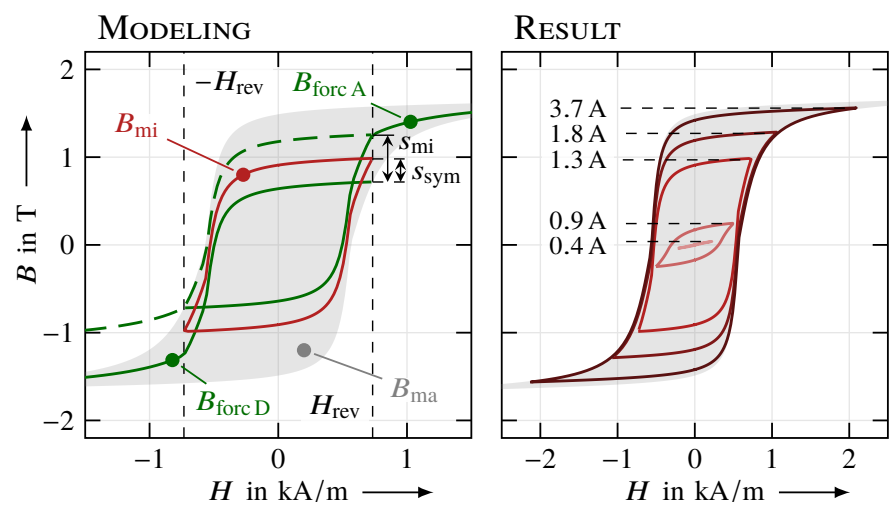

Fig. 6. Modeling of the minor loops and results for various loads $\hat{I}$ for a toroidal core with $d_{\text {outer }}=125 \mathrm{~mm}$ and $d_{\text {inner }}=85 \mathrm{~mm}$ and a circular cross-section.

As seen in Fig. 6, the resulting final minor loop can therefore be expressed analytically for any load point as:

$$
B_{\mathrm{mi}}\left(-H_{\mathrm{rev}} \leq H \leq H_{\mathrm{rev}}\right)=\left\{\begin{array}{l}
B_{\text {forc } \mathrm{A}}(H)-s_{\mathrm{sym}} \\
B_{\text {forc } \mathrm{D}}(H)+s_{\mathrm{sym}}
\end{array}\right.
$$

\section{Dynamic Hysteresis Model}

The basis of modeling the frequency dependent behavior of an iron core rests upon the fact that the magnetization process exhibits a certain delay between the applied field and the resulting flux density. This relationship was derived analytically in the frequency domain for thin laminated sheets by Stoll [11] who proposed to modify the common material property $B=\mu H$ to include a hysteresis angle $\vartheta_{\text {hyst }}$ :

$$
\underline{B}=\mu_{0} \mu_{\mathrm{r}} \underline{H} \mathrm{e}^{-\mathrm{j} \vartheta_{\text {hyst }}}, \quad \text { so } \quad \underline{\mu}_{\text {hyst }}=\mu_{0} \mu_{\mathrm{r}} \mathrm{e}^{-\mathrm{j} \vartheta_{\text {hyst }}} .
$$

The complex hysteresis permeability $\mu_{\text {hyst }}$ can substitute the linear permeability $\mu$ in any core mode $\bar{l}$. Our example reluctance from (4) therefore transforms to:

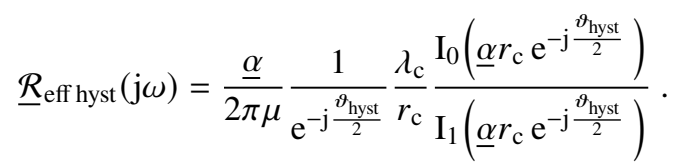

Using the minor loop derivation of this article and the concept of equivalent ellipses presented by Cardelli [22] and Rodriguez et al. [28] it is possible to arrive at a value for the load point dependent phase lag or hysteresis angle $\vartheta_{\text {hyst }}$.

\section{A. Equivalent Ellipse}

The equivalent ellipse with the semi-major axis $p$ and the semi-minor axis $q$ can be formed by two boundary conditions stated by Cardelli et al. [22]. First, the area enclosed by the ellipse $A_{\text {ell }}$ equals the area enclosed by the minor loop:

$$
A_{\text {ell }}=\pi B_{\text {max }} H_{\text {max }} \sin \left(\vartheta_{\text {hyst }}\right)=A_{\text {mi }}=\oint B_{\text {mi }} \mathrm{d} H .
$$

Second, the vertices of the ellipse need to equal that of the minor loop:

$$
B_{\max }=B_{\text {forc A }}\left(H_{\text {rev }}\right)-s_{\text {sym }}=B_{\text {rev }} \quad \text { and } \quad H_{\text {max }}=H_{\text {rev }} .
$$
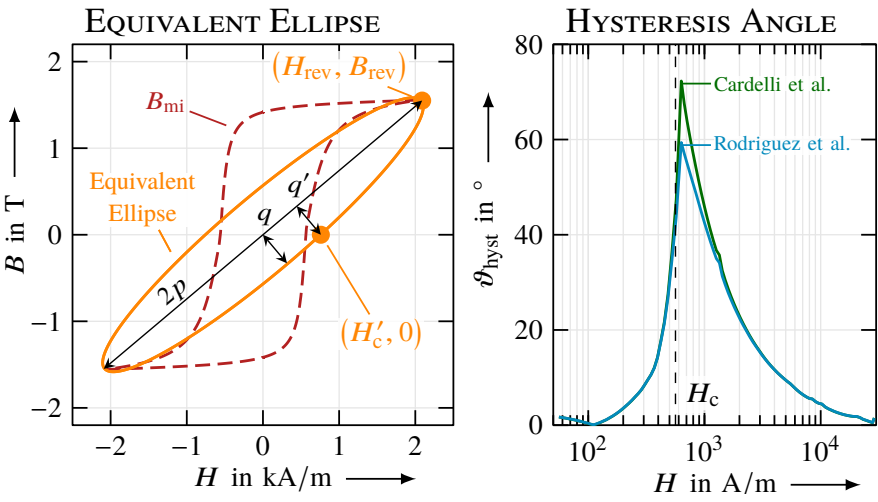

Fig. 7. a) Minor Loop and its equivalent ellipse, b) Comparison between the Cardelli [22] and Rodriguez [28] model for the phase lag dependent on the applied external magnetic field strength

These two conditions ensure that the hysteresis loss is equal for the ellipse as well as the hysteresis loop as shown in Fig. 7a. Solving (24) yields the desired expression

$$
\vartheta_{\text {hyst }}=\arcsin \frac{A_{\mathrm{mi}}}{\pi B_{\max } H_{\mathrm{max}}} .
$$

Since the minor loop modeling is able to determine the hysteresis loop for any load point, it is also possible to calculate the phase lag for a large range of applied fields (Fig. 7b).

Rodriguez et al. [28] proposed an alternative approach for determining the phase lag from the equivalent ellipse, assuming that $q=q^{\prime}$ as illustrated in Fig. 7a. Here the point where the ellipse crosses the $H$-axis, defined as $H_{c}^{\prime}$, is used so that

$$
\vartheta_{\text {hyst }}=\arcsin \left(H_{\mathrm{c}}^{\prime} / H_{\max }\right) \quad \Rightarrow \quad A_{\mathrm{ell}}=\pi \mu H_{\mathrm{c}}^{\prime} H_{\max } .
$$

This assumption is fairly accurate for materials with narrow hysteresis loops and holds true over a wide range of field strengths, where both models show similar results (Fig. 7b). For materials with significantly wider hysteresis loops, like in our case, and especially for applied field strengths around $H_{\mathrm{c}}$, where the equivalent ellipse is more circular, a clear deviation of about $20 \%$ can be observed for the peak of the curve. That is why we prefer Cardelli's definition in (26). Furthermore, the sharp peak demands a more thorough discussion. It is unclear whether this is the result of the mathematical modeling, an inherent characteristic of the employed $15 \mathrm{NiCr} 13$ steel or general behavior for ferromagnetic materials. Regardless of the reasoning, extensive measurements in this critical range of field strengths with different materials should be undertaken, if they have not already been done, unknown to the authors' knowledge. However, the non-monotonic relationship is expectable, as the phase lag heavily depends on the angle of the semi-major axis of the ellipse and therefore $B_{\max }$. These, in turn, are dependent on the differential permeability $\mu_{\mathrm{vir}}(H)$ of the virgin magnetization curve, which is not monotonic either.

\section{B. Frequency Domain Model}

Once the phase lag for a load point $\vartheta_{\text {hyst }}\left(H_{\text {rev }}\right)$ is known, it can be used in conjunction with $\underline{\mathcal{R}}_{\text {eff hyst }}(\mathrm{j} \omega)$ from (23) to complete a frequency domain analysis, where the load dependent permeability $\mu_{\mathrm{rrev}}=B_{\mathrm{rev}} /\left(\mu_{0} H_{\text {rev }}\right)$ is used to model 


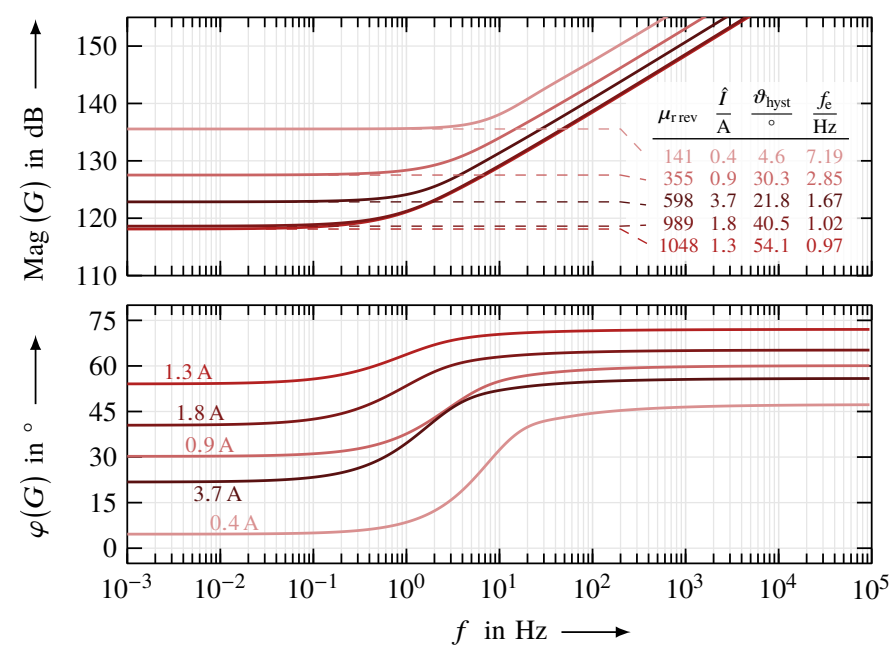

Fig. 8. Frequency response for the effective reluctance $\mathcal{R}_{\text {eff hyst }}$ including hysteresis for various loads $\hat{I}$ given the geometry of [15]

the influence of saturation. Naturally, the stationary value $\mathcal{R}_{0 \text { hyst }}$ as well as the eddy current edge frequency $f_{\mathrm{e}}$ are significantly altered by this values [1]. In Fig. 8 it becomes apparent, that according to their reciprocal proportionality against $\mu_{\mathrm{rrev}}$, both values decrease for increasing loads $\hat{I}$, until more or less the coercive field $H_{\mathrm{c}}$ is reached, where the virgin curve flattens out. For higher loads $\mathcal{R}_{0 \text { hyst }}$ and $f_{\mathrm{e}}$ increase again.

The phase response exhibits a low frequency value which equals that of $\vartheta_{\text {hyst }}\left(H_{\text {rev }}\right)$ and a high frequency value limited to $\vartheta_{\text {emax }}+\vartheta_{\text {hyst }}\left(H_{\text {rev }}\right) / 2$. Both of which were also observed by Rodriguez et al. [28]. The maximum eddy current phase lag $\vartheta_{\mathrm{e} \text { max }}=45^{\circ}$, which is caused by the magnetic skin effect, exhibits the half-order system behavior, we already discussed in [1]. As a consequence, if we omit the eddy currents, we can conclude that the impact of the hysteresis in solid cores is actually easing for higher frequencies, which we will further elaborate in the following section V-C.

\section{Dynamic Hysteresis Loops}

It is well-known, that hysteresis loops are widening for higher frequencies [30] and in this article we propose a new method to model these dynamic hysteresis loops. For the lack of adequate measurement equipment, we cannot verify it, though. On the other hand, an FE-analysis would be misleading, as the field distribution in a nonlaminated core is not homogeneous due to the magnetic skin effect. So different parts of the core exhibit not just varying permeabilities, but also different hysteresis angles. For that reason, FE-analyses are not able to model hysteresis phenomena realistically or at least the authors are not aware of any commercially available FE-software, which is able to employ load-dependent hysteresis angles or minor loops ${ }^{1}$.

In the last section V-B we established a description of the overall phase lag $\varphi$ of an electromagnetic core, by the means of its effective reluctance $\underline{\mathcal{R}}_{\text {eff hyst }}$. By substituting the hysteresis

${ }^{1}$ Software available to the authors: ANSYS MAXwELL 2020 allows hysteresis only in time-domain. FEMm 4.2 employs the simplified linear relation $\theta_{\text {hyst }}=$ $\theta_{\text {hyst } \max } \cdot \mu / \mu_{\max }$, which appears to be inconclusive.

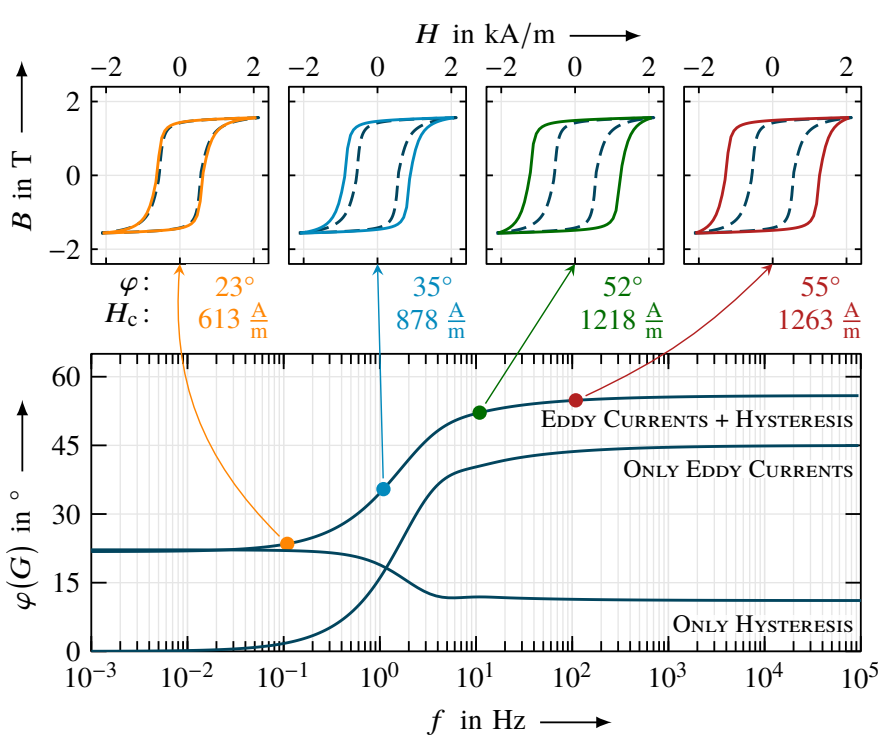

Fig. 9. Dynamic hysteresis loops based on the phase response for the effective reluctance $\mathcal{R}_{\text {eff hyst }}$ for $\hat{I}=3.7 \mathrm{~A}, \mu_{\text {r rev }}=598, \vartheta_{\text {hyst }}=21.8^{\circ}$

angle $\vartheta_{\text {hyst }}$ in (26) with $\varphi$, we obtain a description of the dynamic zero-crossing of the equivalent ellipse:

$$
H_{\mathrm{c}}^{\prime}(f)=H_{\max } \sin (\varphi) \text { with } H_{\mathrm{c} 0}^{\prime}=H_{\mathrm{max}} \sin \left(\vartheta_{\text {hyst }}\right) .
$$

It can be translated to the dynamic coercive field strength

$$
H_{\mathrm{c}}(f)=\frac{H_{\max }}{H_{\mathrm{c} 0}^{\prime}} \sin (\varphi) \cdot H_{\mathrm{c}}=\frac{\sin (\varphi)}{\sin \left(\vartheta_{\text {hyst }}\right)} \cdot H_{\mathrm{c}} .
$$

By inserting (29) into (9) and (10), respectively, we get the frequency-dependent amplitude factor $a(f)$

$$
a(f)=\frac{B_{\max }-c \cdot H_{\max }}{\arctan \left(b \cdot\left(H_{\max }-H_{\mathrm{c}}(f)\right)\right)} .
$$

Subsequently, we can model any hysteresis loop for a given load and frequency as illustrated in Fig. 9. As expected, the hysteresis loop widens for an increasing frequency up to $100 \mathrm{~Hz}$, when the overall phase lag $\varphi$ of the system remains steady. We already observed in section V-B and Fig. 8, that the greater part of the phase lag $\varphi_{\text {eddy }}$ stems from the eddy currents. If we regard the hysteresis lag $\varphi_{\text {hyst }}=\vartheta_{\text {hyst }}(f)$ isolated from the eddy currents, it becomes obvious that the impact of hysteresis alone actually decreases for higher frequencies, as long as no air gap is involved. We conjecture, that this may be a general property of soft magnetic materials, but leave the proof to future studies. However, hysteresis and eddy currents will always occur in combination, so the widening of hysteresis loops is not put into question.

\section{Fractional-Order All-Pass Hysteresis Filter}

In [1] we showed, that it is possible to model the overall effective reluctance $\mathcal{R}_{\text {eff }}$ without hysteresis in a rational form, in such a way that it can be discretized and implemented as a filter-cascade in a real-time control system. However, considering the hysteresis with $\vartheta_{\text {hyst }}$ leads to the highly nonlinear transcendental system $\underline{\mathcal{R}}_{\text {eff hyst }}$ in (23), for which no analytical rational approximation exists. Hence, we have to 
split the system into the already known eddy current part $\mathcal{R}_{\text {eff }}$ and a pure hysteresis filter $G_{\text {hyst }}$ as demonstrated in Fig. 9. As the amplitude response of the system is practically not affected by the hysteresis and $\varphi\left(G_{\text {hyst }}\right)$ is $\varphi_{\text {hyst }}$, we can identify an all-pass-behavior. A conventional all-pass with the exponent $\gamma=1$, here in the Laplace-domain,

$$
G_{\mathrm{AP}}^{\mathrm{LF}}(s)=\frac{(1 \mp s T)^{\gamma}}{(1 \pm s T)^{\gamma}} \quad \text { or } \quad G_{\mathrm{AP}}^{\mathrm{HF}}(s)=\frac{(s T \mp 1)^{\gamma}}{(s T \pm 1)^{\gamma}}
$$

either starts at $\varphi=0^{\circ}$ for low frequencies and changes to $\mp 180^{\circ}$ (LF) or behaves the other way around (HF), in which the phase shift $\Delta \varphi=\gamma \pi=\pi$ stems from $\gamma=1$. The hysteresis demands an all-pass with a phase response between the angles $\varphi_{1}=\vartheta_{\text {hyst }}$ and $\varphi_{2}=\vartheta_{\text {hyst }} / 2$ (Fig. 9), though ${ }^{2}$. Consequently, the exponents $\gamma_{1 \mid 2}=\varphi_{1 \mid 2} / \pi$ are fractions, implying a fractional-order system (FO-system [33]). Furthermore, as neither the start nor the end value equals $\varphi=0^{\circ}$ a series connection of two all-pass filters is needed. A logical first proposition is the following $\mathrm{HF}-\mathrm{FO}-$ all-pass filter

$$
G_{\text {hyst }}(s)=\frac{\left(s T_{\mathrm{e}}-1\right)^{\gamma_{1}-\gamma_{2}}}{\left(s T_{\mathrm{e}}+1\right)^{\gamma_{1}-\gamma_{2}}} \cdot \frac{\left(s T_{2}-1\right)^{\gamma_{2}}}{\left(s T_{2}+1\right)^{\gamma_{2}}} \text { with } T_{2} \ll T_{\mathrm{e}},
$$

where the term with $T_{\mathrm{e}}$ models the phase drop around the eddy current edge frequency $f_{\mathrm{e}}=1 /\left(2 \pi T_{\mathrm{e}}\right)$ and the second term with $T_{2}$ is a simple phase offset with a cut off frequency $f_{2} \gg f_{\mathrm{e}}$ somewhere above our range of interest. For a practical implementation fractional-order systems have to be transformed into a rational form and one of the most suitable approaches for an implicit FO-system, like in this case, is the implicit Matsuda approximation (IMA), as thoroughly discussed in [1]. Yet it requires a zero-phase for the stationary case. For that reason, we propose the alternative LF-FO-all-pass filter

$$
G_{\text {hyst }}(s)=\frac{\left(1+s T_{1}\right)^{\gamma_{1}}}{\left(1-s T_{1}\right)^{\gamma_{1}}} \cdot \frac{\left(1-s T_{\mathrm{e}}\right)^{\gamma_{1}-\gamma_{2}}}{\left(1+s T_{\mathrm{e}}\right)^{\gamma_{1}-\gamma_{2}}} \text { with } T_{1} \gg T_{\mathrm{e}},
$$

first offsetting the phase by the starting angle $\varphi_{1}$ from zero at a frequency $f_{1}=1 /\left(2 \pi T_{1}\right)$ as low as possible. Around $f_{\mathrm{e}}$ the phase shift is reduced by $\varphi_{1}-\varphi_{2}$ to $\vartheta_{\text {hyst }} / 2$. This filter can by approximated by the IMA and implemented as a filter-cascade on a digital control system.

As the IMA has to be applied to every single of the four binomial terms $(1+s T)^{\gamma}$ in (33) separately, and each requires an approximation order of at least $m=9$, as recommended in [1], the total filter order $m_{\Sigma \text { hyst }}=4 m-2=34$ is very high. The more important eddy current filter or the flux density estimator $G_{\mathrm{FE}}(s)$ from (3) employs a filter order of $m_{\Sigma \text { eddy }}=9 \ldots 51$ (cf. [1]) itself, so it is questionable whether both filters can be applied at the same time. Furthermore, the hysteresis filter $G_{\text {hyst }}(s)$ as stated here, is not load-dependent. Every parameter change that influences $\vartheta_{\text {hyst }}$, like a varying permeability e.g. within a look-up table, demand a recalculation of the filters coefficients, which undoubtedly cannot be done in real-time.

\footnotetext{
${ }^{2}$ In case an air gap is part of the magnetic circuit, the starting angle $\varphi_{1}$ has to be corrected by the ratio of the stationary core-only reluctance $\mathcal{R}_{0 \text { core }}$ and total reluctance $\mathcal{R}_{0 \text { tot }}$, the latter including the air gap: $\varphi_{1}=\vartheta_{\text {hyst }} \cdot \mathcal{R}_{0 \text { core }} / \mathcal{R}_{0 \text { tot }}$ This may leads to $\varphi_{1}<\varphi_{2}$.
}

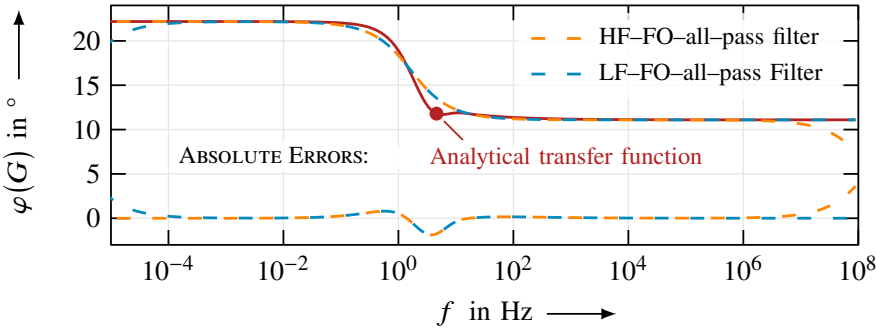

Fig. 10. Hysteresis-only phase lag $\varphi_{\text {hyst }}$ in comparison with the low-frequency and high-frequency fractional-order all-pass filter

\section{E. Discussion of the impact the nonlinearities}

We regard it as impossible, to consider hysteresis and varying permeabilities in a frequency domain model at the same time. Hence, we recommend to not employ a hysteresis compensation in real-time actuator control systems, which raises the question, which impact it actually has on the system behavior. For frequencies above the eddy current edge frequency $f>f_{\mathrm{e}}$ the influence of the hysteresis is widely exceeded by the eddy currents and a possibly inaccurate determination of the loaddependent permeability $\mu$. For $f<f_{\mathrm{e}}$ it depends on the main field time constant $T_{\mathrm{h}}=L_{\mathrm{h}} / R_{\mathrm{Cu}}=1 /\left(2 \pi f_{\mathrm{h}}\right)$ : in the case that $f_{\mathrm{h}}>f_{\mathrm{e}}$, which tends to apply to nonlaminated actuators as studied here, the coil resistance $R_{\mathrm{Cu}}$ is dominant up to $f_{\mathrm{h}}$. That is why we consider it valid to neglect the hysteresis in our setup. It remains the case $f_{\mathrm{h}}<f_{\mathrm{e}}$ in the range $f_{\mathrm{h}}<f<f_{\mathrm{e}}$ that exists mainly for laminated cores, for which the high phase lag, as illustrated in Fig. 9, can have a significant impact. Especially in rather inductive systems like power or instrument transformers, which usually operate on less variable loads and rely on more linear core materials, our improved hysteresis model may is a valuable addition to existing models. In all other cases, the hysteresis filter is still suited to facilitate the controller design of the inner flux density control (Fig. 1) by means of a worst-case load scenario. It allows a more accurate modeling of the controlled system and therefore provides an estimate of an additional phase margin one should consider for improved stability.

The limitations of the hysteresis filter in respect to its loaddependency also applies to the proposed flux density estimator. We consider it impossible to implement e. g. look-up tables, as the estimator coefficients could not be updated in real-time. Hence, we are restricted to the choice of a constant permeability $\mu$ for the underlying model. However, first measurements on a magnetic thrust bearing, whose results we will publish soon, have shown that the highly nonlinear magnetization curve of the deployed $15 \mathrm{NiCr} 13$ steel influences the dynamic of the proposed flux density control to a great extend. We observed, that the exact determination of the permeability $\mu$, corresponding to the applied load, is crucial to achieve an optimal system behavior. But it became apparent that in conjunction with the magnetic skin effect it is impossible to reliably predict the best matching permeability for all operating points of the actuator. That is why we decided for our case to assume the knee point flux density $B_{\mathrm{k}}$ (cf. Fig. $3, \mu_{\mathrm{r}}=880$ ) as worst-case load, as it leads to a rather moderate flux density control with presumably increased stability margins. 


\section{Static Fringing and LeaKage Flux Model}

Unlike hysteresis and saturation, fringing fluxes $\Phi^{v}$ and leakage fluxes $\Phi^{\sigma}$ are a more tangible perturbation phenomenon and therefore more commonly subject of investigation in actuator modeling. For the sake of readability we speak of non-core fluxes $\Phi^{v \mid \sigma}$ covering both flux types as opposed to the core fluxes or main path fluxes exclusively permeating through the iron core and the geometrical air gap ${ }^{3}$. Early adaptations of reluctance networks considering non-core fluxes in radial magnetic bearings go back to Meeker [34] and were later applied to thrust bearings by [35], [36] and also combined bearings [37], [38], like in our case, but with significantly differing leakage paths and sometimes only for the magnetostatic case.

Non-core fluxes in laminated radial bearings and conventional rotating machines are usually subject to only minor fluctuations and can be considered by a constant leakage $k_{\sigma}$ and fringing factor $k_{v}$, respectively. In nonlaminated thrust bearings the magnetic skin effect alters the flux paths and the overall impact of the non-core fluxes depends on the frequency. For this reasons, we study the potential and the practicability of a reluctance network and check whether it can be implemented within the flux density estimator.

As a first step we assume a symmetrical thrust bearing with an omitted bias flux, as shown in Fig. 11, to simplify the identification of the non-core flux paths in the field results of a magnetostatic FE-analysis. Otherwise, negligible but misleading asymmetrical compensation fluxes occur. After identifying the reluctance network we calculate quantitative values for the non-core reluctances $\mathcal{R}^{v \mid \sigma}$. To achieve this, we use the fluxes determined for every network branch by a magnetostatic FE-analysis for a given magnetomotive force (mmf) $\Theta$. In section VII, the same network can then be used backwards to calculate the dynamic flux distribution for various frequencies. Furthermore, we perform the transition to the real asymmetrical thrust bearing in section VII-B.

Generally, in thrust bearings we can differ between fringing and leakage fluxes as well as passive non-core fluxes. The latter have no influence on the thrust force, as they do not bypass any air gaps. On the other hand, fringing fluxes bypass a single air gap in the direction of the force and therefore contribute to it. Leakage fluxes permeate perpendicular to the force or bypass both air gaps and are not part of the force generating main flux $\Phi_{\mathrm{h}}$. The following core $\Phi$, fringing $\Phi^{v}$ and leakage fluxes $\Phi^{\sigma}$ can be identified unambiguously from the magnetostatic FE-analysis in Fig. 11:

\section{a) Core and Air Gap Fluxes}

$\Phi_{\text {ao }}$ - closes main flux path outside of coil in the outer stator and defines the total flux $\Phi_{\mathrm{t}}$ quantitatively.

$\Phi_{\mathrm{giL} \mid \mathrm{R}}$ - cross the air gaps in the main flux path. They are the major force-generating fluxes.

$\Phi_{\mathrm{di}}-$ crosses the disk and rotor and equals the force-generating main flux, after subtraction of $\Phi_{\mathrm{dc}}^{\sigma}$.

${ }^{3}$ The air gap fluxes $\Phi_{\text {gi }}$, which directly cross the air gap without bulging, are attributed to the core fluxes, as they share a comparable frequency behaviour.

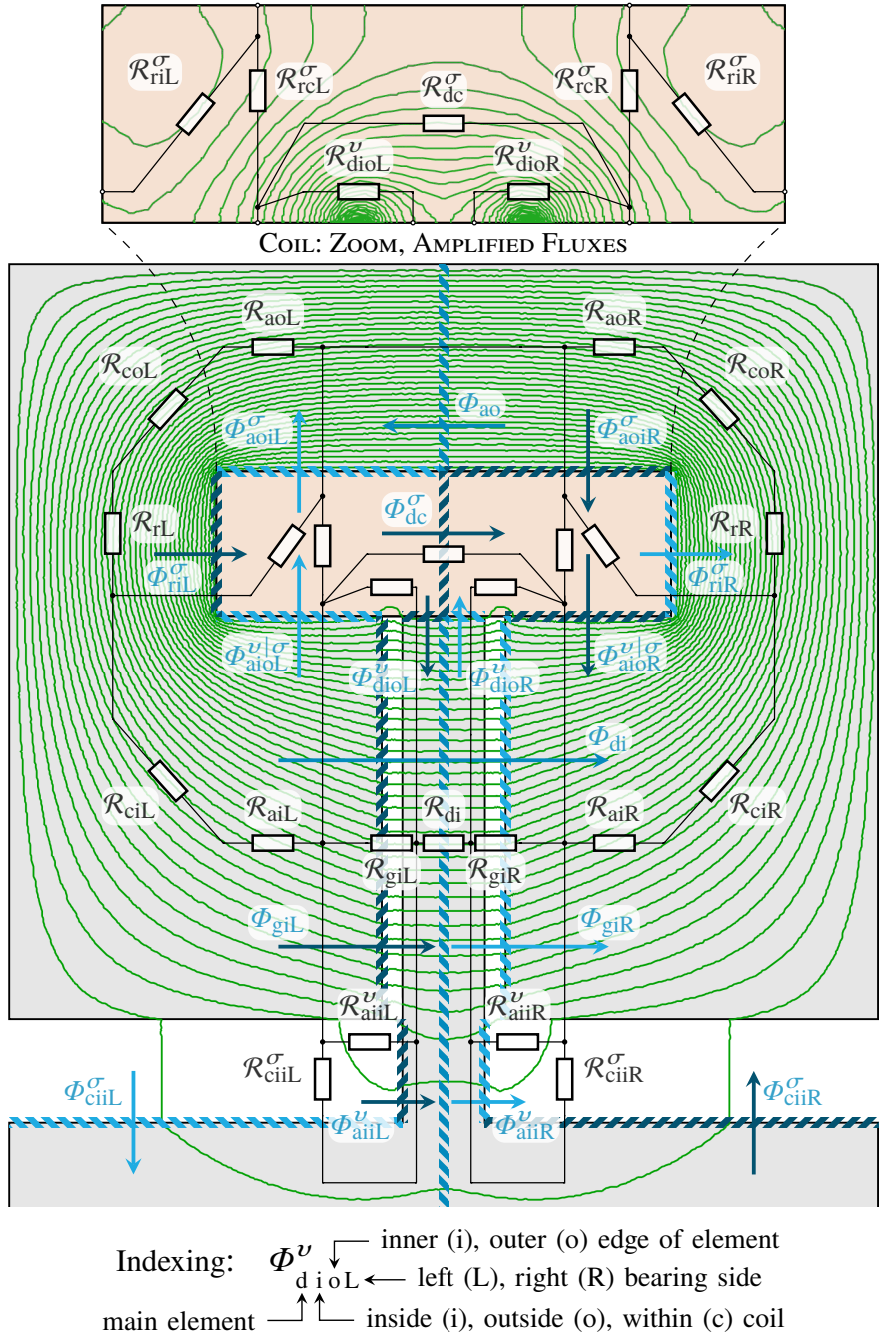

Fig. 11. Magnetostatic flux distribution of the symmetrical magnetic thrust bearing with omitted bias flux: Identification of fringing $\Phi^{v}$ and leakage fluxes $\Phi^{\sigma}$ and reluctances from linear FE-analysis

\section{b) Fringing Fluxes $\Phi^{v}$}

$\Phi_{\text {dioL } \mid \mathrm{R}}^{v}$ - bypass single coil-sided air gap

$\Phi_{\text {aiil } \mid \mathrm{R}}^{v}$ - bypass single shaft-sided air gap.

c) Leakage Fluxes $\Phi^{\sigma}$

$\Phi_{\text {ciiL } \mid \mathrm{R}}^{\sigma}$ - bypass between single air gap and shaft, perpendicular to thrust force. They vanish for high frequencies.

$\Phi_{\mathrm{dc}}^{\sigma}$ - bypasses whole disk and therefore both air gaps.

$\Phi_{\text {riL } \mid \mathrm{R}}^{\sigma}$ - leak from the inner side of the radial core elements $\mathcal{R}_{\mathrm{rL} \mid \mathrm{R}}$. They form negligible flux swirls, which alter their shape even for small changes of frequency, load or symmetry. To simplify the calculation of the network, they are neglected.

\section{d) Passive non-core Fluxes}

$\Phi_{\mathrm{rcL} \mid \mathrm{R}}^{\sigma}$ - cross the coil through its center. Depending on the symmetry, they form leakage swirls only for low frequencies. For high frequencies they change direction and exclusively bypass core elements with no direct impact on the force.

Every one of these non-core fluxes $\Phi^{v \mid \sigma}$ is assigned to a noncore reluctance $\mathcal{R}^{v \mid \sigma}$. In combination with the core reluctances $\mathcal{R}$ in Fig. 2 (calculated in [1]) we obtain the complete reluctance network in Fig. 12. 


$$
\mathcal{R}=\left[\begin{array}{cccccccccc}
\mathcal{R}_{\mathrm{soL}}+\mathcal{R}_{\mathrm{rcL}}^{\sigma} & 0 & 0 & 0 & 0 & 0 & 0 & 0 & -\mathcal{R}_{\mathrm{rcL}}^{\sigma} & 0 \\
0 & \mathcal{R}_{\mathrm{soR}}+\mathcal{R}_{\mathrm{rcR}}^{\sigma} & 0 & 0 & 0 & 0 & 0 & 0 & -\mathcal{R}_{\mathrm{rcR}}^{\sigma} & 0 \\
0 & 0 & \mathcal{R}_{\mathrm{ciiL}}^{\sigma}+\mathcal{R}_{\mathrm{aiiL}}^{v} & 0 & -\mathcal{R}_{\mathrm{aiiL}}^{v} & 0 & 0 & 0 & 0 & 0 \\
0 & 0 & 0 & \mathcal{R}_{\mathrm{ciiR}}^{\sigma}+\mathcal{R}_{\mathrm{aiiR}}^{v} & 0 & -\mathcal{R}_{\mathrm{aiiR}}^{v} & 0 & 0 & 0 & 0 \\
0 & 0 & -\mathcal{R}_{\mathrm{aiiL}}^{v} & 0 & \mathcal{R}_{\mathrm{giL}}+\mathcal{R}_{\mathrm{aiiL}}^{v} & 0 & -\mathcal{R}_{\mathrm{giL}} & 0 & 0 & 0 \\
0 & 0 & 0 & -\mathcal{R}_{\mathrm{aiiR}}^{v} & 0 & \mathcal{R}_{\mathrm{giR}}+\mathcal{R}_{\mathrm{aii}}^{v} & 0 & -\mathcal{R}_{\mathrm{giR}} & 0 & 0 \\
0 & 0 & 0 & 0 & -\mathcal{R}_{\mathrm{giL}} & 0 & \mathcal{R}_{\mathrm{giL}}+\mathcal{R}_{\mathrm{dioL}}^{v} & 0 & 0 & -\mathcal{R}_{\mathrm{dioL}}^{v} \\
0 & 0 & 0 & 0 & 0 & -\mathcal{R}_{\mathrm{giR}} & 0 & \mathcal{R}_{\mathrm{giR}}+\mathcal{R}_{\mathrm{dioR}}^{v} & 0 & -\mathcal{R}_{\mathrm{dioR}}^{v} \\
-\mathcal{R}_{\mathrm{rcL}}^{\sigma} & -\mathcal{R}_{\mathrm{rcR}}^{\sigma} & 0 & 0 & 0 & 0 & 0 & 0 & \mathcal{R}_{\mathrm{dc}}^{\sigma}+\mathcal{R}_{\mathrm{rcL}}^{\sigma}+\mathcal{R}_{\mathrm{rcR}}^{\sigma} & -\mathcal{R}_{\mathrm{dc}}^{\sigma} \\
0 & 0 & 0 & 0 & 0 & 0 & -\mathcal{R}_{\mathrm{dioL}}^{v} & -\mathcal{R}_{\mathrm{dioR}}^{v} & -\mathcal{R}_{\mathrm{dc}}^{\sigma} & \mathcal{R}_{\mathrm{di}}+\mathcal{R}_{\mathrm{dc}}^{\sigma}+\mathcal{R}_{\mathrm{dioL}}^{v}+\mathcal{R}_{\mathrm{dioR}}^{v}
\end{array}\right]
$$

with $\mathcal{R}_{\mathrm{soL} \mid \mathrm{R}}=\mathcal{R}_{\mathrm{aoL} \mid \mathrm{R}}+\mathcal{R}_{\mathrm{coL} \mid \mathrm{R}}+\mathcal{R}_{\mathrm{rL} \mid \mathrm{R}}+\mathcal{R}_{\mathrm{ciL} \mid \mathrm{R}}+\mathcal{R}_{\mathrm{aiL} \mid \mathrm{R}}$

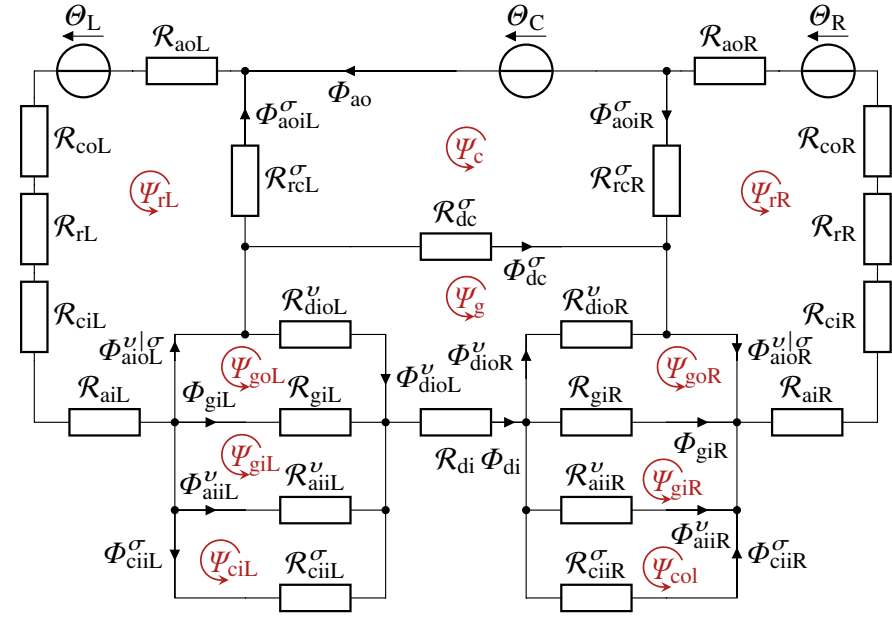

Fig. 12. Complete reluctance network including fringing $\mathcal{R}_{i}^{v}$ and leakage reluctances $\mathcal{R}_{i}^{\sigma}$

Physically, the $\operatorname{mmf} \Theta$ is a distributed quantity and should be considered by a multitude of sources in the network. However, since the exact distribution is not known, it would increase the number of unknowns and the network could not be solved. Rather we place the mmf where the total magnetic flux $\Phi_{\mathrm{t}}$ is at its maximum: in the outer stator yoke. To be able to model the swirls $\Phi_{\mathrm{rcL} \mid \mathrm{R}}^{\sigma}=\Phi_{\mathrm{aoiL} \mid \mathrm{R}}^{\sigma}$ (with $\Phi_{\mathrm{riL} / \mathrm{R}}^{\sigma}$ neglected), we distribute $\Theta$ to the outer corners and the center of the stator (Fig. 12). However, to consider the changing directions of the fluxes $\Phi_{\mathrm{aoiL} \mid \mathrm{R}}^{\sigma}$ and to keep the network solvable, the manually chosen weighting has to be adapted for the static and dynamic as well as symmetric and asymmetric case.

We remind that in magnetic circuits a mesh is defined as $\sum \Theta_{i}=\sum \mathcal{R}_{i} \Phi_{i}$, so the sum of mmf sources equals the sum of magnetic voltage drops $\sum \mathcal{R}_{i} \Phi_{i}$. Hence, the reluctance network is described by the system of linear equations

$$
\mathcal{R} \cdot \boldsymbol{\Psi}=\boldsymbol{\Theta}
$$

with the reluctance matrix $\mathcal{R}$ as well as the vectors $\boldsymbol{\Psi}$ and $\boldsymbol{\Theta}$ describing the flux linkages of every mesh and the $\mathrm{mmf}$ sources, respectively. The flux linkages

$$
\boldsymbol{\Psi}=\left[\begin{array}{llllllllll}
\Psi_{\mathrm{rL}} & \Psi_{\mathrm{rR}} & \Psi_{\mathrm{cil}} & \Psi_{\mathrm{ciR}} & \Psi_{\mathrm{giL}} & \Psi_{\mathrm{giR}} & \Psi_{\mathrm{goL}} & \Psi_{\mathrm{goR}} & \Psi_{\mathrm{c}} & \Psi_{\mathrm{g}}
\end{array}\right]^{\mathrm{T}}
$$

are calculated step-wise using Kirchhoff's circuit laws and the fluxes determined from the magnetostatic $\mathrm{FE}$-analysis (as indicated in Fig. 11:

$$
\begin{aligned}
\Psi_{\mathrm{rL}} & =\Phi_{\mathrm{ao}}+\Phi_{\mathrm{aoiL}}^{\sigma} & \Psi_{\mathrm{giL}} & =\Phi_{\mathrm{aiiL}}^{v}+\Phi_{\mathrm{ciiL}}^{\sigma} \\
\Psi_{\mathrm{rR}} & =\Phi_{\mathrm{ao}}+\Phi_{\mathrm{aoiR}}^{\sigma} & \Psi_{\mathrm{giR}} & =\Phi_{\mathrm{aiiR}}^{v}+\Phi_{\mathrm{ciiR}}^{\sigma} \\
\Psi_{\mathrm{ciL}} & =\Phi_{\mathrm{ciil}}^{\sigma} & \Psi_{\mathrm{goL}} & =\Phi_{\mathrm{ao}}-\Phi_{\mathrm{dc}}^{\sigma}-\Phi_{\mathrm{dio}}^{v} \\
\Psi_{\mathrm{ciR}} & =\Phi_{\mathrm{ciiR}}^{\sigma} & \Psi_{\mathrm{goR}} & =\Phi_{\mathrm{ao}}-\Phi_{\mathrm{dc}}^{\sigma}-\Phi_{\mathrm{dio}}^{v} \\
\Psi_{\mathrm{c}} & =\Phi_{\mathrm{ao}} & \Psi_{\mathrm{g}} & =\Phi_{\mathrm{ao}}-\Phi_{\mathrm{dc}}^{\sigma}
\end{aligned}
$$

With more equations the system would be overdetermined, so the focus lies on the total flux $\Phi_{\mathrm{ao}}=\Phi_{\mathrm{t}}$ which can be quantified exactly and defines the reference flux. For the static and symmetric case the mmf sources are distributed equally to the stator corners, to maintain the swirl character of $\Phi_{\text {aoiL } \mid \mathrm{R}}^{\sigma}$ :

$$
\Theta_{\mathrm{mL}}=\Theta_{\mathrm{R}}=\frac{\Theta}{2} \quad \text { and } \quad \Theta_{\mathrm{C}}=0,
$$

leading to the vector

$$
\boldsymbol{\Theta}=\left[\begin{array}{llllllllll}
\Theta_{\mathrm{L}} & \Theta_{\mathrm{R}} & 0 & 0 & 0 & 0 & 0 & 0 & \Theta_{\mathrm{C}} & 0
\end{array}\right]^{\mathrm{T}} .
$$

The reluctance matrix $\mathcal{R}$ in (39) with $\mathcal{R}_{\mathrm{soL} \mid \mathrm{R}}=\mathcal{R}_{\mathrm{aoL} \mid \mathrm{R}}+\mathcal{R}_{\mathrm{coL} \mid \mathrm{R}}+$ $\mathcal{R}_{\mathrm{rL} \mid \mathrm{R}}+\mathcal{R}_{\mathrm{ciL} \mid \mathrm{R}}+\mathcal{R}_{\mathrm{aiL} \mid \mathrm{R}}$ is composed of the sought-after noncore reluctances $\mathcal{R}_{i}^{v \mid \sigma}$ as well as the known analytical solutions for the static part reluctances $\mathcal{R}_{0 i}$ from Fig. 2 [1]. To solve the equation system for all $\mathcal{R}_{i}^{v \mid \sigma}$ e.g. the Gaussian elimination algorithm or a computer algebra system (CAS) can be used. At this point we omit the last mesh equation for $\Psi_{\mathrm{g}}$, as there are only nine unknown reluctances, but it is later needed for the backward calculation of the leakage fluxes from the network.

The quantitative values of the determined non-core reluctances $\mathcal{R}_{i}^{v \mid \sigma}$ are summarized in Table III and compared with the parallel core reluctances $\mathcal{R}_{\|}$they bypass. It becomes apparent, that in the operational bandwidth of the thrust bearing (up to $1 \mathrm{kHz}$ ) the central leakage reluctances $\mathcal{R}_{\mathrm{rcL} \mid \mathrm{R}}^{\sigma}$ have the least impact and can be neglected. On the other hand, a comparison of Table III with Fig. 13 shows that the high impact of the reluctances $\mathcal{R}_{\text {aiiL } \mid \mathrm{R}}^{v}$ and $\mathcal{R}_{\text {ciil } \mid \mathrm{R}}^{\sigma}$ on the side of the shaft, with $7.8 \%$ and $14 \%$, respectively, is misleading. The actual fluxes $\Phi_{\text {aii } \mid \mathrm{R}}^{v}$ and $\Phi_{\text {ciil } \mid \mathrm{R}}^{\sigma}$ fall below $1 \%$ for more than $1 \mathrm{kHz}$, due to the magnetic skin effect. This discrepancy can not be compensated by the reluctance network, as we will show later (cf. Fig. 18). 
TABLE III

FRINGING $\mathcal{R}_{i}^{v}$ AND LEAKAGE RELUCTANCES $\mathcal{R}_{i}^{\sigma}$ DETERMINED BY FE-ANALYSIS IN COMPARISON WITH BYPASSED CORE RELUCTANCES $\mathcal{R}_{\|}$FOR THE SYMMETRIC CASE FOR $0 \mathrm{~Hz}$ AND $1 \mathrm{kHz}$

\begin{tabular}{|c|c|c|c|c|c|}
\hline $\mathcal{R}_{i}^{v \mid \sigma}$ & in $\mathrm{A} / \mathrm{Vs}$ & $\mathcal{R}_{\|}$ & in $\mathrm{A} / \mathrm{Vs}$ & \multicolumn{2}{|c|}{$\mathcal{R}_{\|} / \mathcal{R}_{i}^{v \mid \sigma} \mathcal{R}_{\|} / \mathcal{R}_{i}^{v \mid \sigma}$} \\
\hline \multicolumn{4}{|c|}{ SYMMETRIC CASE: } & $0 \mathrm{~Hz}$ & $1 \mathrm{kHz}$ \\
\hline $\mathcal{R}_{\mathrm{rcL} \mid \mathrm{R}}^{\sigma}$ & $2.36 \cdot 10^{7}$ & $\mathcal{R}_{\mathrm{soL} \mid \mathrm{R}}{ }^{*}$ & $1.08 \cdot 10^{4}$ & $0.05 \%$ & $2.06 \%$ \\
\hline $\mathcal{R}_{\mathrm{dioL} \mid \mathrm{R}}^{v}$ & $5.94 \cdot 10^{6}$ & $\mathcal{R}_{\mathrm{giL} \mid \mathrm{R}}$ & $1.01 \cdot 10^{5}$ & $2.37 \%$ & $6.22 \%$ \\
\hline $\mathcal{R}_{\text {aiiL } \mid \mathrm{R}}^{v}$ & $3.39 \cdot 10^{6}$ & $\mathcal{R}_{\mathrm{giL} \mid \mathrm{R}}$ & $1.01 \cdot 10^{5}$ & $2.99 \%$ & $7.84 \%$ \\
\hline $\mathcal{R}_{\mathrm{ciiL} \mid \mathrm{R}}^{\sigma}$ & $1.89 \cdot 10^{6}$ & $\mathcal{R}_{\mathrm{giL} \mid \mathrm{R}}$ & $1.01 \cdot 10^{5}$ & $5.36 \%$ & $14.06 \%$ \\
\hline $\mathcal{R}_{\mathrm{dc}}^{\sigma}$ & $1.51 \cdot 10^{7}$ & $\mathcal{R}_{\mathrm{di}}+\mathcal{R}_{\mathrm{giL}}+\mathcal{R}_{\mathrm{giR}}$ & $2.03 \cdot 10^{5}$ & $1.34 \%$ & $4.18 \%$ \\
\hline
\end{tabular}

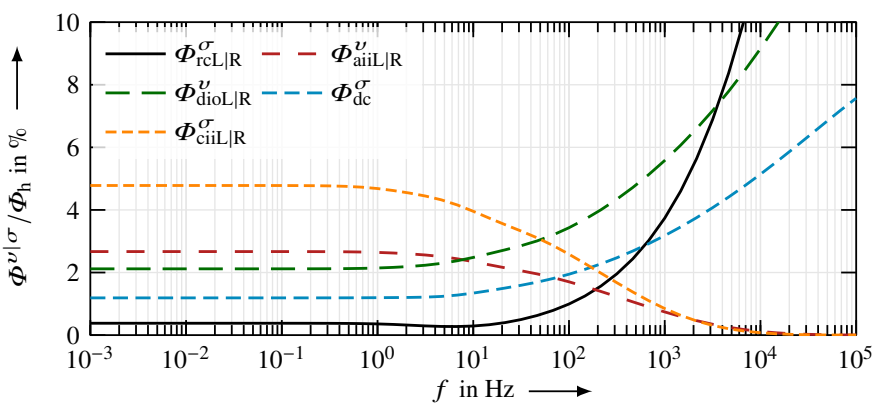

Fig. 13. Share of fringing $\Phi^{v}$ and leakage fluxes $\Phi^{\sigma}$ on the total flux $\Phi_{\mathrm{t}}=\Phi_{\mathrm{ao}}$ for the symmetric thrust bearing

\section{Dynamic Fringing and Leakage Flux Model}

Already for frequencies below $1 \mathrm{~Hz}$ the magnetic skin effect causes the core fluxes to permeate closer to the coil. As a consequence, the leakage flux $\Phi_{\mathrm{rcL} \mid \mathrm{R}}^{\sigma}$ change their direction, so the mmf-source has to be arranged centrally in the outer branch of the reluctance network in Fig. 12:

$$
\Theta_{\mathrm{L}}=\Theta_{\mathrm{R}}=0 \quad \text { and } \quad \Theta_{\mathrm{C}}=\Theta
$$

In the fully computed reluctance network from section VI, we can now substitute the static part reluctances $\mathcal{R}_{0 i}$ by the frequency-dependent, and therefore complex, effective part reluctances $\underline{\mathcal{R}}_{i}$ [1]. For a given magnetic voltage $\Theta$ it is then possible to calculate the mesh flux linkages $\underline{\boldsymbol{\Psi}}$ and hence every core flux $\underline{\Phi}_{i}$ and non-core flux $\underline{\Phi}^{v \mid \sigma}$ for any frequency. This way, the sole analytical overall effective reluctance $\mathcal{R}_{\text {eff }}$ can be corrected to the leakage- and fringing-accounting effective reluctance

$$
\underline{\mathcal{R}}_{\mathrm{eff} \mathrm{t}}^{\prime}=\frac{\Theta}{\underline{\Phi}_{\mathrm{ao}}}=\frac{\Theta}{\underline{\Phi}_{\mathrm{t}}},
$$

which we compare with a dynamic FE-analysis. Fig. 14 shows, that the amplitude error of $\underline{\mathcal{R}}_{\text {eff }}(\mathrm{ca} .1 \mathrm{~dB})$ toward the FE simulation results can be eliminated almost entirely by the reluctance network. The leakage and fringing fluxes are also responsible for a considerable decay of the phase from its theoretical limit of $45^{\circ}$ for high frequencies, which is describable by the network as well. Only the modeling error of the original analytical solution around the eddy current edge frequency $f_{\mathrm{e}}$ (cf. [1]) persists.

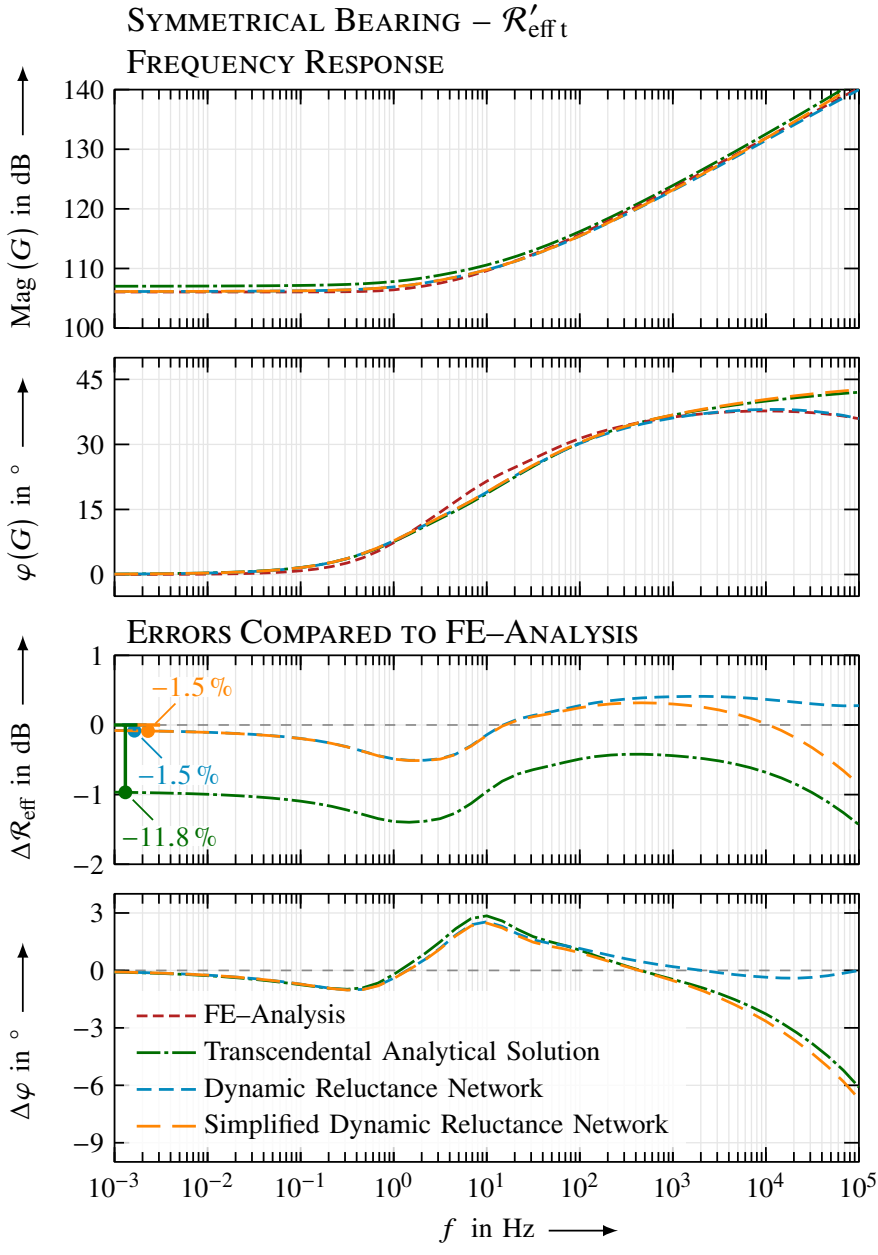

Fig. 14. Frequency responses for the corrected total effective reluctance $\mathcal{R}_{\text {eff }}^{\prime}$ relating to the total flux $\Phi_{\mathrm{t}}$ and its absolute magnitude- and phase errors in respect to the reference $\mathrm{FE}$-analysis

\section{A. Simplified dynamic reluctance network}

The complexity of the complete reluctance network (Fig. 12) leads to a significant increase in the system order $m_{\Sigma}$ of the approximated, rational variant of $\underline{\mathcal{R}}_{\text {eff }}$ (cf. [1]) and is therefore not applicable for the flux density estimator $G_{\mathrm{FS}}$. Hence, we propose the following simplifications, based on Table III, Fig. 13 and the qualitative evaluation of the finite element field distribution.

- The fringing fluxes $\underline{\Phi}_{\text {dioL } \mid \mathrm{R}}^{v}$ and $\underline{\Phi}_{\text {aii } \mid \mathrm{R}}^{v}$ and their corresponding reluctances are combined and assigned to the air gap fringing reluctances $\mathcal{R}_{\mathrm{giL} \mid \mathrm{R}}^{v}$ :

$$
\mathcal{R}_{\text {giL } \mid \mathrm{R}}^{v}=\frac{\mathcal{R}_{\mathrm{dio} \mid \mathrm{R}}^{v} \mathcal{R}_{\text {aii } \mid \mathrm{R}}^{v}}{\mathcal{R}_{\mathrm{dio} \mid \mathrm{R}}^{v}+\mathcal{R}_{\text {aii } \mid \mathrm{R}}^{v}} .
$$

- The central leakage reluctance $\mathcal{R}_{\mathrm{dc}}^{\sigma}$, bypassing both air gaps, is divided into two equal parts and assigned to a single air gap each. Together with the leakage reluctance $\mathcal{R}_{\text {ciil } \mid \mathrm{R}}^{\sigma}$ on the shaft side, they form the air gap leakage reluctances $\mathcal{R}_{\mathrm{giL} \mid \mathrm{R}}^{\sigma}$ :

$$
\mathcal{R}_{\mathrm{giL} \mid \mathrm{R}}^{\sigma}=\frac{\frac{1}{2} \mathcal{R}_{\mathrm{dc}}^{\sigma} \mathcal{R}_{\mathrm{cii} \mid \mathrm{R}}^{\sigma}}{\frac{1}{2} \mathcal{R}_{\mathrm{dc}}^{\sigma}+\mathcal{R}_{\mathrm{cil} \mid \mathrm{R}}^{\sigma}} .
$$


- To determine the corrected total effective reluctance $\mathcal{R}_{\text {eff t }}^{\prime}$, relating to the total flux $\underline{\Phi}_{\mathrm{ao}}=\underline{\Phi}_{\mathrm{t}}$ and not considering the force, we merge $\mathcal{R}_{\text {giL|R }}^{v}$ and $\mathcal{R}_{\text {giL } \mid \mathrm{R}}^{\sigma}$ to the combined air gap fringing and leakage reluctance $\mathcal{R}_{\mathrm{giL} \mid \mathrm{R}}^{v \sigma}$ :

$$
\mathcal{R}_{\mathrm{giL} \mid \mathrm{R}}^{v \sigma}=\frac{\mathcal{R}_{\mathrm{giL} \mid \mathrm{R}}^{v} \mathcal{R}_{\mathrm{giL} \mid \mathrm{R}}^{\sigma}}{\mathcal{R}_{\mathrm{giL} \mid \mathrm{R}}^{v}+\mathcal{R}_{\mathrm{giL} \mid \mathrm{R}}^{\sigma}} .
$$

- The respective "geometrical" air gap reluctances in the main flux path are equal for both bearing sides, independently from the symmetry, so that $\underline{\mathcal{R}}_{\mathrm{giL} \mid \mathrm{R}}=\underline{\mathcal{R}}_{\mathrm{gi}}$. Furthermore we calculate the arithmetic mean of $\mathcal{R}_{\mathrm{giL} \mid \mathrm{R}}^{\overline{v \sigma}}$ to obtain the single corrected air gap reluctance:

$$
\underline{\mathcal{R}}_{\mathrm{gi}}^{\prime}=2\left(\overline{\mathcal{R}}_{\mathrm{gi}}^{v \sigma} \| \underline{\mathcal{R}}_{\mathrm{gi}}\right) \text { with } \overline{\mathcal{R}}_{\mathrm{gi}}^{v \sigma}=\frac{1}{2}\left(\mathcal{R}_{\mathrm{giL}}^{v \sigma}+\mathcal{R}_{\mathrm{giR}}^{v \sigma}\right) \text {. }
$$

The introduced error of $\mathcal{R}_{\mathrm{gi}}^{\prime}$ compared to $\underline{\mathcal{R}}_{\mathrm{giL} \mid \mathrm{R}} \| \mathcal{R}_{\mathrm{giL} \mid \mathrm{R}}^{v \sigma}$ is only $0.03 \%$, which is why we adopt this step for

$$
\overline{\mathcal{R}}_{\mathrm{gi}}^{v}=\frac{1}{2}\left(\mathcal{R}_{\mathrm{giL}}^{v}+\mathcal{R}_{\mathrm{giR}}^{v}\right), \quad \overline{\mathcal{R}}_{\mathrm{gi}}^{\sigma}=\frac{1}{2}\left(\mathcal{R}_{\mathrm{giL}}^{\sigma}+\mathcal{R}_{\mathrm{giR}}^{\sigma}\right) .
$$

as well.

- The outer axial core reluctances $\mathcal{R}_{\mathrm{aoL} \mid \mathrm{R}}$ are summed up to a single reluctance $\underline{\mathcal{R}}_{\text {ao }}$ by addition of their characteristic lengths.

- As a last step we neglect the transversal reluctances $\mathcal{R}_{\mathrm{rcL} \mid \mathrm{R}}^{\sigma}$, since their impact for low frequencies is negligible (cf. Fig. 13). Although in the dynamic case for high frequencies above $1 \mathrm{kHz}$ the respective fluxes $\Phi_{\mathrm{rL} \mid \mathrm{R}}^{\sigma}$ are dominant, the impact of $\mathcal{R}_{\mathrm{rcL} \mid \mathrm{R}}^{\sigma}$ on the magnitude response of $\mathcal{R}_{\mathrm{efft}}^{\prime}$ is limited due its passive characteristic. However, the previously observed phase decay for frequencies above $1 \mathrm{kHz}$ cannot be modeled without $\mathcal{R}_{\mathrm{rcL} \mid \mathrm{R}}^{\sigma}$, which is deemed uncritical, as it lies outside of the bearings operating bandwidth. If higher bandwidths require the consideration of $\mathcal{R}_{\mathrm{rcL} \mid \mathrm{R}}^{\sigma}$, one should be aware that the system order $m_{\Sigma}$ almost doubles.

As result we obtain the simplified dynamic reluctance network in Fig. 15, which forms the leakage- and fringingaccounting total effective reluctance $\underline{\mathcal{R}}_{\mathrm{efft}}^{\prime}=\Theta / \underline{\Phi}_{\mathrm{t}}$ by summing up of its part reluctances. Up to ca. $100 \mathrm{~Hz}$ both, the full and the simplified network, lead to identical results. In the further relevant bandwidth up to $1 \mathrm{kHz}$ the magnitude and phase error remains low with $<0.4 \mathrm{~dB}$ and $<1^{\circ}$, respectively (cf. Fig. 14).

However, in this form the network cannot just yet be applied to the flux density estimator, as the magnetic bearing control relies on the force-generating main flux $\underline{\Phi}_{\mathrm{h}}=\underline{\Phi}_{\mathrm{f}}=\underline{\Phi}_{\mathrm{gi}}+\underline{\Phi}^{v}$ and not the total flux $\underline{\Phi}_{\mathrm{t}}$ as illustrated in Fig. 16. Consequentially, the force-related total effective reluctance $\mathcal{R}_{\text {eff f }}^{\prime}=\Theta / \underline{\Phi}_{\text {f }}$ only refers to the force-generating flux $\underline{\Phi}_{\mathrm{f}}$. The results for the accordingly adapted $\underline{\mathcal{R}}_{\text {eff f }}^{\prime}$ will be shown in section VII-D.

\section{B. Transition to Asymmetric Bearing}

In the asymmetric real thrust bearing the position of the coil was shifted to the right as depicted in Fig. 2. We took this measure to equalize the static reluctances of the bias flux paths and therefore enable equal forces in both air gaps. In terms of fringing and leakage fluxes, we observe that they are facilitated for high frequencies $f>1 \mathrm{kHz}$ especially on the left

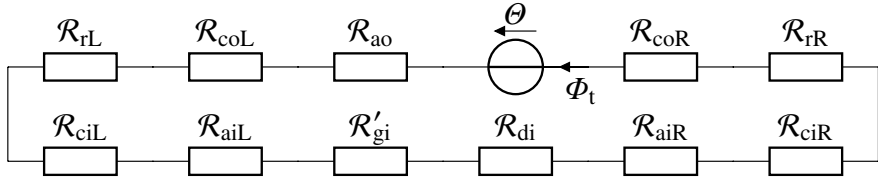

Fig. 15. Simplified dynamic reluctance network with corrected air gap reluctance $\mathcal{R}_{\mathrm{gi}}^{\prime}$

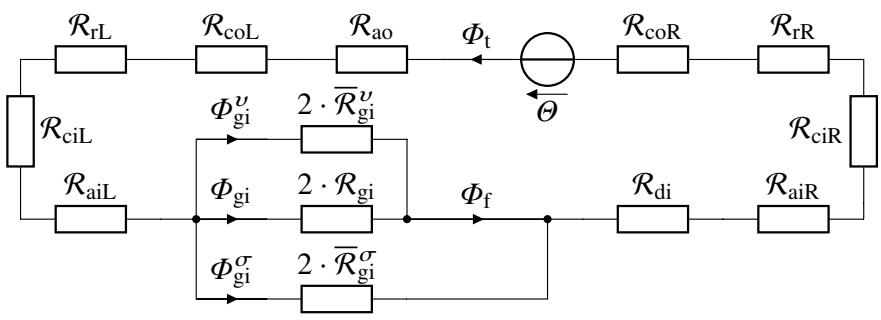

Fig. 16. Simplified dynamic reluctance network with total flux path split into the force-generating fringing part $\Phi^{v}$ and leakage part $\Phi^{\sigma}$

TABLE IV

FRINGING $\mathcal{R}_{i}^{v}$ AND LEAKAGE RELUCTANCES $\mathcal{R}_{i}^{\sigma}$ DETERMINED By FE-ANALYSIS IN

\begin{tabular}{|c|c|c|c|c|c|}
\hline $\mathcal{R}_{i}^{v \mid \sigma}$ & in $\mathrm{A} / \mathrm{Vs}$ & $\mathcal{R}_{\|}$ & in $\mathrm{A} / \mathrm{Vs}$ & \multicolumn{2}{|c|}{ 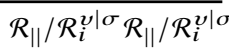 } \\
\hline \multicolumn{3}{|c|}{ ASYMmetric CASE: } & & $0 \mathrm{~Hz}$ & $1 \mathrm{kHz}$ \\
\hline $\mathcal{R}_{\mathrm{rcL}}^{\sigma}$ & $1.02 \cdot 10^{6}$ & $\mathcal{R}_{\mathrm{SoL}}$ & $7.93 \cdot 10^{3}$ & $0.78 \%$ & $31.43 \%$ \\
\hline $\mathcal{R}_{\mathrm{rcR}}^{\sigma}$ & $6.95 \cdot 10^{6}$ & $\mathcal{R}_{\mathrm{SoR}}$ & $1.47 \cdot 10^{4}$ & $0.21 \%$ & $9.36 \%$ \\
\hline $\mathcal{R}_{\mathrm{dioL}}^{v}$ & $3.48 \cdot 10^{6}$ & $\mathcal{R}_{\mathrm{giL}}$ & $1.02 \cdot 10^{5}$ & $3.65 \%$ & $19.29 \%$ \\
\hline $\mathcal{R}_{\mathrm{dioR}}^{v}$ & $5.42 \cdot 10^{6}$ & $\mathcal{R}_{\mathrm{giR}}$ & $1.02 \cdot 10^{5}$ & $2.59 \%$ & $14.77 \%$ \\
\hline $\mathcal{R}_{\mathrm{aiiL}}^{v}$ & $2.72 \cdot 10^{6}$ & $\mathcal{R}_{\mathrm{giL}}$ & $1.02 \cdot 10^{5}$ & $3.79 \%$ & $9.82 \%$ \\
\hline $\mathcal{R}_{\mathrm{aiiR}}^{v}$ & $3.33 \cdot 10^{6}$ & $\mathcal{R}_{\mathrm{giR}}$ & $1.02 \cdot 10^{5}$ & $3.10 \%$ & $8.04 \%$ \\
\hline $\mathcal{R}_{\mathrm{ciiL}}^{\sigma}$ & $4.33 \cdot 10^{6}$ & $\mathcal{R}_{\mathrm{giL}}$ & $1.02 \cdot 10^{5}$ & $2.38 \%$ & $11.20 \%$ \\
\hline $\mathcal{R}_{\text {ciiR }}^{\sigma}$ & $1.88 \cdot 10^{6}$ & $\mathcal{R}_{\mathrm{giR}}$ & $1.02 \cdot 10^{5}$ & $5.48 \%$ & $19.24 \%$ \\
\hline $\mathcal{R}_{\mathrm{dc}}^{\sigma}$ & $1.06 \cdot 10^{7}$ & $\mathcal{R}_{\mathrm{di}}+\mathcal{R}_{\mathrm{giL}}+\mathcal{R}_{\mathrm{giR}}$ & $2.06 \cdot 10^{5}$ & $1.95 \%$ & $5.98 \%$ \\
\hline
\end{tabular}
COMPARISON WITH BYPASSED CORE RELUCTANCES $\mathcal{R}_{\|}$FOR THE ASYMMETRIC CASE FOR $0 \mathrm{~Hz}$ AND $1 \mathrm{kHz}$

side of the bearing. It becomes apparent in Table IV, where the ratios $\mathcal{R}_{\|} / \mathcal{R}_{i}^{v \mid \sigma}$ for left-sided reluctances are considerably higher than in the symmetric case. Furthermore, the right-sided central leakage flux $\underline{\Phi}_{\text {aoir }}^{\sigma}$ remains a swirl, as in Fig. 12, over the entire frequency range, while its left-sided counterpart $\Phi_{\text {aoil }}^{\sigma}$ is always passive (it changes its direction compared to Fig. 12). To account for this asymmetry, the magnetic voltage source has to be placed in the outer right corner of the network:

$$
\Theta_{\mathrm{L}}=\Theta_{\mathrm{C}}=0 \quad \text { and } \quad \Theta_{\mathrm{R}}=\Theta .
$$

By this slight adjustment, the full and the simplified reluctance network enables comparable results for the corrected total effective reluctance $\underline{\mathcal{R}}_{\text {eff }}^{\prime}$ like in the symmetric case, at least in the bandwidth below $1 \mathrm{kHz}$.

\section{Analytical Calculation of non-core Reluctances}

To verify the FE-analysis or to allow a faster design process, it is of interest to find an analytical description for the leakage reluctances. However, in 1941 Roters [39] already stated that the precise determination of the reluctance of flux paths in air is practically impossible, as they do not follow geometrically simple paths. Especially, since every actuator geometry features individual characteristics and boundary 
a) $180^{\circ}: \mathcal{R}_{\mathrm{dioL} \mid \mathrm{R}}^{v} \mathcal{R}_{\mathrm{dc}}^{\sigma}$

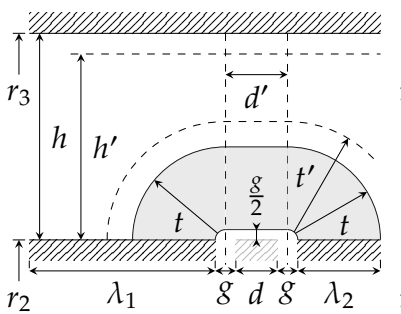

b) $90^{\circ}: \mathcal{R}_{\text {aiiL } \mid \mathrm{R}}^{v}$

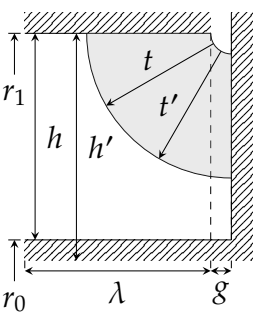

c) $0^{\circ}: \mathcal{R}_{\mathrm{ciiL} \mid \mathrm{R}}^{\sigma}$

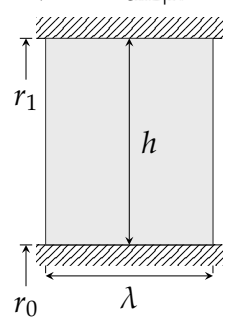

Fig. 17. Definition of analytical leakage/fringing flux path for the a) $180^{\circ}$ reluctances $\mathcal{R}_{\mathrm{dc}}^{\sigma}$ and $\mathcal{R}_{\mathrm{dioL} / \mathrm{R}}^{\nu}$ (for the case $\lambda_{2}<t^{\prime}<\lambda_{1}$ ), for the b) $90^{\circ}-$ reluctances $\mathcal{R}_{\text {aiiL } \mid \mathrm{R}}^{v}$ (for the case $t^{\prime}<\lambda$ ) and for the c) $0^{\circ}$-reluctances $\mathcal{R}_{\text {ciil } \mid \mathrm{R}}^{\sigma}$

conditions which change depending on the applied load and frequency. Previous works studying leakage fluxes in magnetic thrust bearings, like [35] or [37], neglect necessary geometrical and electromagnetic boundary conditions. Besides, they did not verify their findings and we were not able to reproduce them conclusively. Hence we tried to find a more precise analytical description of the leakage reluctances based on the most general approaches in [39]. We still consider it a rough estimate, not substituting the FE-analysis, as various assumptions had to be made. Following the simplifications made in section VII-A, we forgo the modeling of the passive central reluctances $\mathcal{R}_{\mathrm{rcL} \mid \mathrm{R}}^{\sigma}$ and concentrate on the geometrically well-defined and force-related reluctances in Fig. 17.

The analytical derivation of the fringing and leakage reluctances is mainly adapted from [39] starting with his definition of a half annulus (p. 131, Fig. 10 in [39])

$$
\mathcal{R}^{v \mid \sigma}=\frac{\pi}{\mu_{0} l} \frac{1}{\ln \left(1+\frac{2 t}{g}\right)}=\frac{\pi}{\mu_{0} l} \frac{1}{\ln \left(\frac{r_{\mathrm{to}}}{r_{\mathrm{ti}}}\right)}
$$

with the depth $l$, the inner arc radius $r_{\mathrm{ti}}=g / 2$ and the outer arc radius $r_{\text {to }}=g / 2+t$. In case of a cylindrical actuator, the depth $l$ equals the average circumference, considering the enlargement of the cross section of the flux path for increasing radii. The arc height $t$ is limited by the width $\lambda$ of the boundary faces and the distance of opposite core elements (Fig. 17).

\section{Case a) $180^{\circ}$-reluctances: $\mathcal{R}_{\mathrm{dioL} \mid \mathrm{R}}^{v}, \mathcal{R}_{\mathrm{dc}}^{\sigma}$}

The field distribution in Fig. 11 illustrates, that the flux paths pertaining to $\mathcal{R}_{\mathrm{dioL} \mid \mathrm{R}}^{v}$ are semicircular, while $\mathcal{R}_{\mathrm{dc}}^{\sigma}$ is composed of two quarter circles and a quasi-homogeneous middle section (Fig. 17). The middle section can be regarded as a hollow cylinder of the length $d^{\prime}=d+g$, depending on the width of the thrust disk $d$. For the quarter and semicircles of $\mathcal{R}_{\mathrm{dio} \mid \mathrm{R}}^{v}$ as well as $\mathcal{R}_{\mathrm{dc}}^{\sigma}$ we define the inner arc radius as half of the air gap width: $r_{\mathrm{ti}}=g / 2$. Hence, the ansatz derived from (53) is identical for both cases (with $d^{\prime}=0$ for $\mathcal{R}_{\text {dioL } \mid \mathrm{R}}^{v}$ ):

$$
\mathcal{R}_{\mathrm{dioL} \mid \mathrm{R}, \mathrm{dc}}^{v \mid \sigma}=2 \frac{\frac{\pi}{2 \mu_{0} l}}{\ln \left(1+\frac{2 t}{g}\right)}+\underbrace{\frac{\frac{d^{\prime}}{\mu_{0} \pi}}{\left(r_{2}+\frac{g}{2}+t\right)^{2}-\left(r_{2}+\frac{g}{2}\right)^{2}}}_{\text {quarter arc }} .
$$

The arc height $t$ equates to the width of the smaller of both boundary faces, but is limited by the maximum arc height $t^{\prime}$ by $t=\min \left(\lambda_{1}, \lambda_{2}, t^{\prime}\right)$, which describes the outermost flux line, which is just able to draw an arc. Flux lines beyond this boundary line preferably close over the opposite core element. Hence, we state

$$
\pi\left(t^{\prime}+\frac{g}{2}\right)+d^{\prime}=2 h^{\prime} \quad \Rightarrow \quad t^{\prime}=\frac{2}{\pi}\left(h^{\prime}-\frac{d^{\prime}}{2}\right)-\frac{g}{2},
$$

with the effective height $h^{\prime}$ of the leakage area, which corrects the geometrical height $h$ by considering the curvature of the cylindrical geometry:

$$
h^{\prime}=r_{2} \ln \left(\frac{r_{2}+h}{r_{2}}\right) \text {. }
$$

The radius and thereby the circumference $l$ of the medium flux line is calculated according to [39] by the geometric mean of the inner and outer arc radius:

$$
l=2 \pi\left(r_{2}+\sqrt{\frac{g}{2} \cdot\left(\frac{g}{2}+t\right)}\right) .
$$

Case b) $90^{\circ}$-reluctances: $\mathcal{R}_{\text {aii } \mid \mathrm{R}}^{v}$

In contrast to case a), the fringing fluxes $\Phi_{\text {aiil } \mid R}^{v}$ leave the iron core to the inside (Fig. 17), requiring adjustments to the first approach. The ansatz

$$
\mathcal{R}_{\text {aiiL } \mid \mathrm{R}}^{v}=\frac{\pi}{2 \mu_{0} l} \frac{1}{\ln \left(1+\frac{t}{g}\right)}
$$

remains equal to the quarter arc in (54), but the inner arc radius is now defined as $r_{\mathrm{ti}}=g$. Thus, the arc height is

$$
t=\min \left(\lambda, t^{\prime}\right) \quad \text { with } \quad t^{\prime}=\frac{2}{\pi} h^{\prime}-g,
$$

where because of the inverse curvature the effective height

$$
h^{\prime}=r_{1} \ln \left(\frac{r_{1}}{r_{1}-h}\right)
$$

is larger than the geometric height $h$, in this case. According to [39] the medium circumference then becomes

$$
l=2 \pi\left(r_{1}+g-\sqrt{g \cdot(g+t)}\right) .
$$

\section{Case c) $0^{\circ}$-reluctances: $\mathcal{R}_{\text {ciiL } \mid \mathrm{R}}^{\sigma}$}

The calculation for the leakage reluctances $\mathcal{R}_{\text {ciil } \mid \mathrm{R}}^{\sigma}$ is trivial. It can be modeled as a hollow cylinder permeated by a flux in radial direction (Fig. 17). Hence, the ansatz is equal to the radial core reluctance $\mathcal{R}_{\mathrm{r}}$ in [1]:

$$
\mathcal{R}_{\text {ciiL } \mid \mathrm{R}}^{\sigma}=\frac{\ln \left(\frac{r}{r-h}\right)}{2 \pi \mu_{0} \lambda} .
$$

Since the curvature has already been considered in (62), the previously done correction of the geometrical height $h$ is not necessary. 
TABLE V

FRINGING $\mathcal{R}_{i}^{v}$ AND LEAKAGE RELUCTANCES $\mathcal{R}_{i}^{\sigma}$ DETERMINED BY FE-ANALYSIS IN COMPARISON WITH ITS ANALYTICAL ESTIMATES

- Relative ERROR: $\epsilon_{\mathrm{rel}}=\left(\mathcal{R}_{\mathrm{ana}}^{v \mid \sigma}-\mathcal{R}_{\mathrm{fem}}^{v \mid \sigma}\right) / \mathcal{R}_{\mathrm{fem}}^{\nu \mid \sigma}$

\begin{tabular}{|c|c|c|c|c|c|c|}
\hline \multirow[b]{2}{*}{ Symbol } & \multicolumn{3}{|c|}{ Symmetric CASE } & \multicolumn{3}{|c|}{ Asymmetric CASE } \\
\hline & $\frac{\mathcal{R}_{\mathrm{fem}}^{\nu \mid \sigma}}{10^{6} \mathrm{~A} / \mathrm{Vs}}$ & $\frac{\mathcal{R}_{\mathrm{ana}}^{v \mid \sigma}}{10^{6} \mathrm{~A} / \mathrm{Vs}}$ & $\frac{\epsilon_{\mathrm{rel}}}{\%}$ & $\frac{\mathcal{R}_{\mathrm{fem}}^{\nu \mid \sigma}}{10^{6} \mathrm{~A} / \mathrm{Vs}}$ & $\frac{\mathcal{R}_{\text {ana }}^{v \mid \sigma}}{10^{6} \mathrm{~A} / \mathrm{Vs}}$ & $\frac{\epsilon_{\text {rel }}}{\%}$ \\
\hline $\mathcal{R}_{\mathrm{dc}}^{\sigma}$ & 15.06 & 11.72 & -22.2 & 10.58 & 11.72 & 10.7 \\
\hline $\begin{array}{l}\mathcal{R}_{\text {dioL }}^{v} \\
\mathcal{R}_{\text {dioR }}^{v}\end{array}$ & 4.25 & 3.87 & -8.8 & $\begin{array}{l}2.81 \\
3.95\end{array}$ & $\begin{array}{l}3.90 \\
3.90\end{array}$ & $\begin{array}{r}38.8 \\
-1.3\end{array}$ \\
\hline $\begin{array}{l}\mathcal{R}_{\text {aiil }}^{v} \\
\mathcal{R}_{\text {aiiR }}^{v}\end{array}$ & 3.37 & 4.65 & 38.2 & $\begin{array}{l}2.71 \\
3.31\end{array}$ & $\begin{array}{l}3.44 \\
4.65\end{array}$ & $\begin{array}{l}27.0 \\
40.8\end{array}$ \\
\hline $\begin{array}{l}\mathcal{R}_{\text {ciil }}^{\sigma} \\
\mathcal{R}_{\text {ciiR }}^{\sigma}\end{array}$ & 1.88 & 1.77 & -5.9 & $\begin{array}{l}4.30 \\
1.87\end{array}$ & $\begin{array}{l}3.86 \\
1.77\end{array}$ & $\begin{array}{r}-10.3 \\
-5.4\end{array}$ \\
\hline $\begin{array}{l}\mathcal{R}_{\mathrm{giL}}^{v} \\
\mathcal{R}_{\mathrm{giR}}^{v}\end{array}$ & 1.88 & 2.11 & 12.6 & $\begin{array}{l}1.38 \\
1.80\end{array}$ & $\begin{array}{l}1.83 \\
2.12\end{array}$ & $\begin{array}{l}32.5 \\
17.9\end{array}$ \\
\hline $\begin{array}{l}\mathcal{R}_{\mathrm{giL}}^{\sigma} \\
\mathcal{R}_{\mathrm{giR}}^{\sigma}\end{array}$ & 1.50 & 1.36 & -9.6 & $\begin{array}{l}2.37 \\
1.38\end{array}$ & $\begin{array}{l}2.33 \\
1.36\end{array}$ & $\begin{array}{l}-2.0 \\
-1.7\end{array}$ \\
\hline $\begin{array}{l}\mathcal{R}_{\mathrm{giL}}^{v \sigma} \\
\mathcal{R}_{\mathrm{giR}}^{v \sigma}\end{array}$ & 0.83 & 0.83 & -1.0 & $\begin{array}{l}0.87 \\
0.78\end{array}$ & $\begin{array}{l}1.02 \\
0.83\end{array}$ & $\begin{array}{r}17.4 \\
5.9\end{array}$ \\
\hline
\end{tabular}

\section{Results of the analytical calculation}

Analogous to the simulatively determined non-core reluctances in (47) to (49), we can also simplify the analytical reluctances to the fringing and leakage air gap reluctances $\mathcal{R}_{\text {giL } \mid \mathrm{R}}^{v}$ and $\mathcal{R}_{\text {giL } \mid \mathrm{R}}^{\sigma}$ as well as the combined air gap fringing and leakage reluctances $\mathcal{R}_{\text {giL } \mid \mathrm{R}}^{v \sigma}$, respectively. The intermediate results in Table $\mathrm{V}$ differ significantly, with up to $40 \%$, from the simulation results, as the analytical calculation considers leakage paths through air exclusively, which in addition cannot be mapped exactly. On the other hand, the FE-results contain core sections permeated by leakage fluxes, which are not included in the analytical core reluctances. Therefore, they provide a much more accurate image of the real flux distribution.

The high errors in the intermediate results lead to a shift in the leakage flux distribution within the full reluctance network (Fig. 12) from the side of the shaft to the side of the coil. With regards to the simplified network (Fig. 15) most of the errors cancel each other out, so only a $1 \%$ error remains for the combined air gap leakage reluctances $\mathcal{R}_{\text {giL } \mid \mathrm{R}}^{v \sigma}$ in the symmetric case. In the asymmetric case acceptable total errors of $17.4 \%$ and $5.9 \%$ persist.

In spite of the simplifications made in this subsection, leading to a rough estimate of the real flux distribution, Fig. 18 shows, that also the analytically determined fringing and leakage reluctances are applicable for the following implementation within the flux density estimator.

\section{Implementation and Conclusion}

Already in section VII-A we indicated, that for the implementation of the flux density estimator not only the corrected total effective reluctance $\underline{\mathcal{R}}_{\text {eff t }}^{\prime}=\Theta / \underline{\Phi}_{\mathrm{t}}$ is decisive, but also the force-related effective reluctance $\underline{\mathcal{R}}_{\text {eff } \mathrm{f}}^{\prime}=\Theta / \underline{\Phi}_{\mathrm{f}}$. The latter relates solely to the actual force-generating part $\underline{\Phi}_{\mathrm{f}}$ of the total flux $\Phi_{\mathrm{t}}$, which we calculate with the flux divider following Fig. 16:

$$
\underline{\Phi}_{\mathrm{f}}=\frac{\mathcal{R}_{\mathrm{gf}} \| \mathcal{R}_{\mathrm{gi}}^{\sigma}}{\mathcal{R}_{\mathrm{gi}}^{\sigma}} \Phi_{\mathrm{t}}=\frac{\mathcal{R}_{\mathrm{gi}}^{\sigma}}{\mathcal{R}_{\mathrm{gi}}^{\sigma}+\underline{\mathcal{R}}_{\mathrm{gf}}} \underline{\Phi}_{\mathrm{t}}
$$

with

$$
\underline{\mathcal{R}}_{\mathrm{gf}}=2 \cdot \frac{\overline{\mathcal{R}}_{\mathrm{gi}}^{v} \cdot \underline{\mathcal{R}}_{\mathrm{gi}}}{\overline{\mathcal{R}}_{\mathrm{gi}}^{v}+\underline{\mathcal{R}}_{\mathrm{gi}}} \quad \text { and } \quad \mathcal{R}_{\mathrm{gi}}^{\sigma}=2 \cdot \overline{\mathcal{R}}_{\mathrm{gi}}^{\sigma} .
$$

By dividing both sides of (63) through the mmf $\Theta=i \cdot N$ and calculating the reciprocal, we obtain the desired form

$$
\underline{\mathcal{R}}_{\text {eff f }}^{\prime}=\left(1+\frac{\mathcal{R}_{\mathrm{gf}}}{\mathcal{R}_{\mathrm{gi}}^{\sigma}}\right) \cdot \underline{\mathcal{R}}_{\mathrm{eff} \mathrm{t}}^{\prime}
$$

The implementation of the combined air gap leakage reluctances $\mathcal{R}_{\text {giL } \mid \mathrm{R}}^{v \sigma}$, leading to $\mathcal{R}_{\text {eff } t}^{\prime}$, is carried out together with the calculation of the air gap reluctance $\mathcal{R}_{\mathrm{gi}}$ or the air gap element (fractional order $\gamma=1 / 4$ ) of the equivalent implicit system $\underline{\mathcal{R}}_{\text {EIS }}$ (cf. [1], eq. (58)) according to (50). In the next step, we derive the force-related reluctance $\mathcal{R}_{\text {eff f f from (65). }}^{\prime}$

All subsequent calculations affect neither the approximations nor the discretization. Only the system orders $m_{\Sigma}, n_{\Sigma}$ of the approximated overall system (sum of all part reluctances in Fig. 15 or equivalent implicit system [1]) increase - in case of

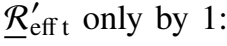

$$
m_{\Sigma \mathrm{t}}=m_{\Sigma}+1, \quad n_{\Sigma \mathrm{t}}=\left(m_{\Sigma}-1\right)+1=m_{\Sigma \mathrm{t}}-1 .
$$

Regarding $\mathcal{R}_{\text {eff f }}^{\prime}$, the flux divider implicates a further increase of the total order by the order $m_{\mathcal{R}_{\mathrm{g}}}$ of the reluctance $\underline{\mathcal{R}}_{\mathrm{gi}}$ :

$$
m_{\Sigma \mathrm{f}}=m_{\Sigma}+m_{\mathcal{R}_{\mathrm{g}}}+1=m_{\Sigma \mathrm{t}}+m_{\mathcal{R}_{\mathrm{g}}}, \quad n_{\Sigma \mathrm{f}}=m_{\Sigma \mathrm{f}}-1 .
$$

However, in [1] we proposed to undertake an additional Padéapproximation (PASR), which overrides the increase in order of $m_{\mathcal{R}_{\mathrm{g}}}=8 \ldots 29$ (for $f<21 \mathrm{kHz}$ ). Therefore, the order of the PASR-solution $m_{\mathcal{P}}$ remains equal to the fringing/leakage-free model. This is a considerable advantage compared to the other proposed approximation method MAEIS, where considering the leakage within $\mathcal{R}_{\text {eff f }}^{\prime}$ increases the total system order by $m_{\mathcal{R}_{\mathrm{g}}}=\left(m_{\Sigma}+1\right) / 2=5 \ldots 25$, limiting the applicability of the approach.

\section{E. Alternative: Constant Correction Factor}

The simplest way to take fringing and leakage fluxes into account, is the introduction of constant correction factors $k_{\mathrm{v \sigma} t}$ and $k_{\mathrm{v \sigma f}}$ (relating to the current $i$ ). Without an increase to the system order, we can correct the total effective reluctance $\underline{\mathcal{R}}_{\text {eff }}$ or inductance $\underline{L}_{\mathrm{eff}}$ according to (4)

$$
\underline{L}_{\mathrm{eff} \mathrm{t}}=k_{\mathrm{v \sigma} \mathrm{t}} N \frac{\underline{\Phi}_{\mathrm{t}}}{i}=\frac{k_{\mathrm{v \sigma \textrm {t }}} N^{2}}{\underline{\mathcal{R}}_{\mathrm{eff}}}=\frac{N^{2}}{\underline{\mathcal{R}}_{\mathrm{eff} \mathrm{t}}^{\prime}} \Rightarrow \underline{\mathcal{R}}_{\mathrm{eff} \mathrm{t}}^{\prime}=\frac{\underline{\mathcal{R}}_{\mathrm{eff}}}{k_{\mathrm{v} \mathrm{t}}} .
$$

The correction factor can be calculated by forming the ratio of the static corrected overall reluctance $\mathcal{R}_{0 \mathrm{t}}^{\prime}$ with the static fringing/leakage-free reluctance $\mathcal{R}_{0}=\mathcal{R}_{\text {eff }}(f=0)$

$$
k_{\mathrm{v \sigma} \mathrm{t}}=\frac{\mathcal{R}_{0}}{\mathcal{R}_{0 \mathrm{t}}^{\prime}}=\frac{\mathcal{R}_{0}}{\Theta / \Phi_{0 \mathrm{t}}},
$$




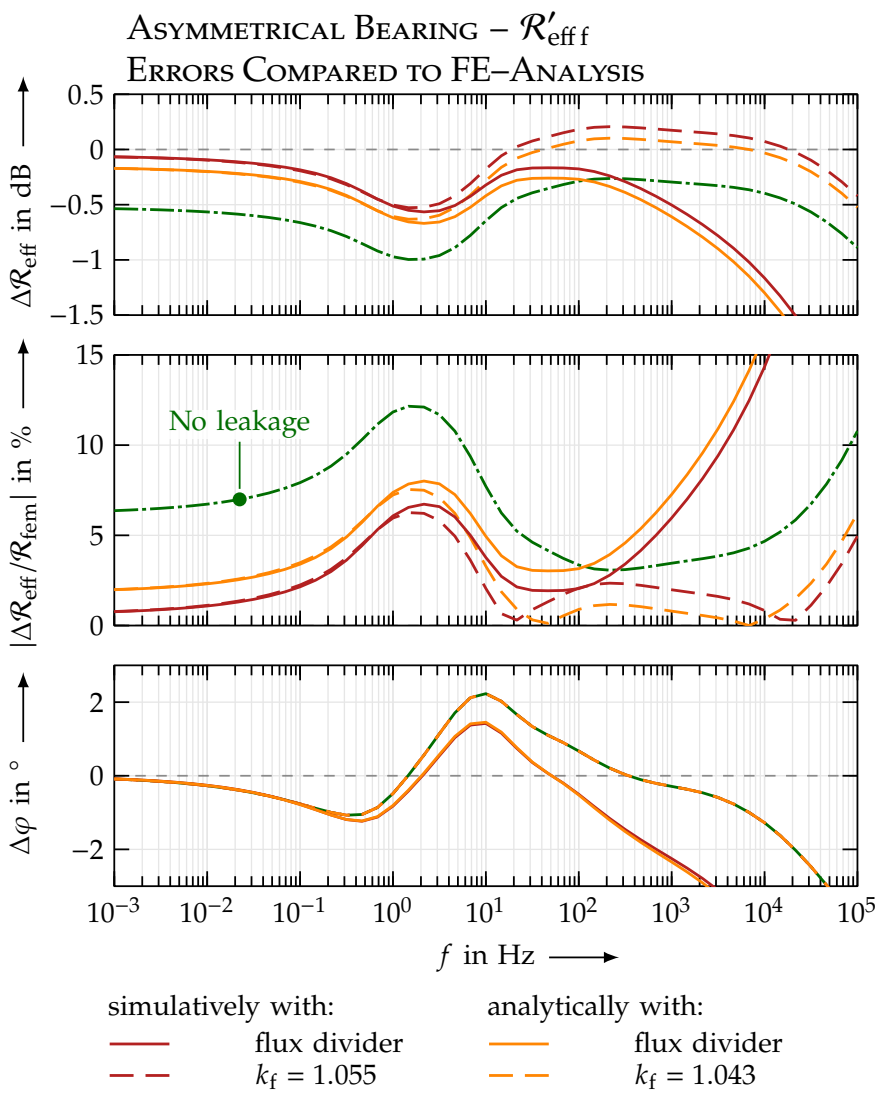

Fig. 18. Absolute and relative magnitude and phase error for the forcegenerating reluctance $\mathcal{R}_{\text {eff f }}^{\prime}$ based on the flux divider (simplified reluctance network) or correction factor $k_{v \sigma \mathrm{f}}$ in respect to reference FEA

where the determination of $\mathcal{R}_{0 \mathrm{t}},(63)$ to (65), or $\Phi_{\mathrm{t}}$ for that matter, can be conducted by analytical calculation or a FEanalysis. By using the flux divider from (63) we determine analogously the force-related effective inductance

$$
\underline{L}_{\text {eff f }}=k_{\text {vof }} N \frac{\Phi_{\mathrm{f}}}{i}=\frac{k_{v \sigma \mathrm{f}} N^{2}}{\underline{\mathcal{R}}_{\text {eff }}}=\frac{N^{2}}{\underline{\mathcal{R}}_{\text {eff f }}^{\prime}} \Rightarrow \underline{\mathcal{R}}_{\text {eff f }}^{\prime}=\frac{\underline{\mathcal{R}}_{\text {eff }}}{k_{\text {vof }}}
$$

and the corresponding correction factor

$$
k_{\mathrm{vof}}=\frac{\mathcal{R}_{0}}{\mathcal{R}_{0 \mathrm{f}}^{\prime}}=\frac{\mathcal{R}_{0}}{\Theta / \Phi_{0 \mathrm{f}}}=\frac{1}{1+\mathcal{R}_{0 \mathrm{gf}} / \mathcal{R}_{\mathrm{gi}}^{\sigma}} \frac{\mathcal{R}_{0}}{\mathcal{R}_{0 \mathrm{t}}^{\prime}} .
$$

\section{F. Comparison}

Up to a frequency of $1 \mathrm{~Hz}$ the implementation of the forcerelated reluctance $\mathcal{R}_{\text {eff f }}^{\prime}$ by means of the flux divider (65) or the correction factor $k_{v \sigma \mathrm{f}}(71)$ leads to equal results, as Fig. 18 illustrates. The absolute error in respect to the $\mathrm{FE}$-analysis can be reduced by $0.35 \mathrm{~dB}$ to $<0.1 \mathrm{~dB}$ (stationary) compared to the fringing/leakage-free $\underline{\mathcal{R}}_{\text {eff }}$, at which point the simulatively determined reluctance is more accurate.

However, as opposed to the corrected total effective reluctance $\mathcal{R}_{\text {eff t }}^{\prime}$ (cf. Fig. 14), the force-related reluctance $\mathcal{R}_{\text {eff f }}^{\prime}$, which is based on the flux divider, does not provide satisfying results in the relevant bandwidth up to $1 \mathrm{kHz}$. The reason for this derives from the the shift in the leakage flux distribution from the shaft to the coil for high frequencies, which cannot be modeled by the reluctance network. Although occurring errors cancel each other out for $\mathcal{R}_{\mathrm{eff}}^{\prime}$, this is not the case for $\mathcal{R}_{\text {eff f }}^{\prime}$ resulting in an inaccurate representation of the forcegenerating flux $\underline{\Phi}_{\mathrm{f}}$. On the other hand, compared to the original analytical solution the correction factor $k_{\mathrm{vo}}$ allows only a slight improvement in accuracy for $1 \mathrm{~Hz}<f<1 \mathrm{kHz}$ (Fig. 18), but more importantly does not degrade it. Hence we conclude, if the force-generating flux $\underline{\Phi}_{\mathrm{f}}$ is to be the focus, the correction factor is the preferred method.

Furthermore, Fig. 18 reveals an unexpected relation. The errors of $\left|\underline{\mathcal{R}}_{\text {eff f }}^{\prime}\right|$ in respect to $\left|\underline{\mathcal{R}}_{\text {eff }}\right|$ are smaller than in the case of $\underline{\mathcal{R}}_{\text {eff t }}^{\prime}$ and remain negative. This in turn means that $\left|\underline{\mathcal{R}}_{\text {eff f }}^{\prime}\right|$ is smaller than $\left|\mathcal{R}_{\text {eff }}\right|$ and the force $f$ is actually increased by the presence of fringing and leakage fluxes. Although, the force-generating flux $\Phi_{\mathrm{f}}$ is indeed smaller than the total flux $\Phi_{\mathrm{t}}$ (reduced by $\Phi^{\sigma}$ ), the fringing and leakage fluxes cause a general reduction of the total reluctance $\left|\underline{\mathcal{R}}_{\text {eff }}^{\prime}\right| \ll\left|\mathcal{R}_{\text {eff }}\right|$ and thus an increase of the actuator force. In our case the latter effect is dominant, resulting in correction factors $k_{\mathrm{v \sigma} t \mid \mathrm{f}}>1$. But it is important to note, that this observation cannot be generalized for all actuators or magnetic bearings and is only probable in case the force-generating fringing fluxes dominate over the leakage fluxes.

\section{G. Correction of Flux Density Estimator}

By recalling the definition of the flux density estimator from (3), we see that there are multiple occurrences of the effective inductance $\underline{L}_{\text {eff }}$ and its stationary counterpart $L_{\mathrm{h}}=$ $\lim _{\omega \rightarrow 0} \underline{L}_{\text {eff }}(\mathrm{j} \omega)$, the main inductance. While the first term is the actual force-related field building component, which we correct with the factor $k_{v \sigma \mathrm{f}}$, the second term describes the magnetizing currents, that relate to the total flux $\underline{\Phi}_{\mathrm{t}}$. Hence, the latter has to be corrected with the factor $k_{v \sigma t}$ finally leading to:

measurable: magnetizing current

$$
\underline{G}_{\mathrm{FE}}^{\prime}(\mathrm{j} \omega)=\frac{\underline{B}_{\text {est }}(\mathrm{j} \omega)}{\underline{i}_{\text {meas }}(\mathrm{j} \omega)}=\underbrace{\frac{L_{\mathrm{hf}}}{N A_{\mathrm{g}}}}_{\text {field }} \cdot \frac{\overbrace{\left.R_{\mathrm{Cu}}+s \underline{L}_{\mathrm{eff}} \mathrm{j} \omega\right)}^{R_{\mathrm{Cu}}+s L_{\mathrm{ht}}}}{\text { force-related: }},
$$

Whether or not this differentiation is appropriate depends on the actuator geometry and the balance between fringing and leakage fluxes. In our case $k_{v \sigma \mathrm{f}}$ and $k_{v \sigma \mathrm{t}}$ differ by considerable $5.2 \%$ and its inclusion comes at no cost.

We note, that in fact the main inductances $L_{\mathrm{ht}}$ and $L_{\mathrm{hf}}$ are not directly affected by the magnetic skin effect, but by the redistribution of the fringing/leakage fluxes. We still assume them to be constant. Theoretically, it would be possible to isolate the frequency-dependent influence of these non-core fluxes and project them on the main inductances as well. However, the improvement would be very low compared to the additional computing effort (cf. section V-E). 


\section{CONCLUSION AND OUTLOOK}

The control of electromagnetic actuators and magnetic bearings usually relies on simplified models and perturbations like eddy currents, hysteresis, saturation, fringing and leakage fluxes are omitted. Especially in case of nonlaminated cores, it is widely accepted that eddy currents have a significant impact on the actuators performance and should be considered within the design of the control. In previous works we laid the groundwork for the first practical digital implementation of a flux density estimator to fully compensate eddy currents in the inner actuator control loop and we will present the first results in the near future.

The considerably minor perturbations hysteresis and saturation as well as fringing and leakage remain solely subjects of academic research. Although various studies exist, they are usually limited to the analytical modeling and FE-analyses of the actuator. For the first time, we discussed the actual applicability of the known analytical models to control design and further improved them.

In the first part of this article we developed a fractional-order all-pass hysteresis filter in the frequency domain, which can model the hysteresis for a fixed load point and an assumed homogeneous field distribution. While these may be valid assumptions for laminated cores and especially for actuators and machines operating in steady-state, it is rarely the case for high-dynamic nonlaminated actuators and magnetic thrust bearings. In this latter case, hysteresis models are indeed limited to evaluation and cannot be applied to control design in a useful manner. However, it is possible to use the hysteresis filter to identify worst-case scenarios and consider an appropriate phase margin to design a stable controller under all conditions.

We found, that varying permeabilities have significant impact on the behavior of the proposed flux density control, but we consider it impossible to include them in the flux density estimator. Hence, we recommend a deliberate choice of $\mu_{\mathrm{r}}$ depending on the maximum load point.

Our study regarding the fringing and leakage fluxes in a magnetic thrust bearing shows, that the practicality of a reluctance network highly depends on whether the total flux $\Phi_{\mathrm{t}}$ and its respective corrected total reluctance $\underline{\mathcal{R}}_{\mathrm{eff} t}^{\prime}$ is of interest or the actual force-generating flux $\underline{\Phi}_{\mathrm{f}}$ relating to $\underline{\mathcal{R}}_{\mathrm{eff}}^{\prime}$ f. In the former case, the proposed networks can significantly reduce model errors (static: $11.8 \%$ ) by 10 percentage points over a wide range of frequencies. The full reluctance network is only beneficial over the simplified network for less relevant frequencies above $1 \mathrm{kHz}$, where the characteristic phase drop is reproduced. In the bandwidth of interest below $1 \mathrm{kHz}$ both networks are equally accurate. In the latter case, due to the magnetic skin effect, neither the full nor the simplified reluctance network can model the changing flux distribution for frequencies above $1 \mathrm{~Hz}$, leading to an incorrect representation of the force-generating flux $\underline{\Phi}_{\mathrm{f}}$ and its according reluctance $\underline{\mathcal{R}}_{\text {eff f }}$. However, a constant correction factors, possibly greater than 1 , reduce the model errors (static: $6.4 \%$ ) by satisfying 5.6 percentage points. Since they do not increase the system order and are most simple to implement we deem them to be the preferred variant for the flux density estimator.
We conclude, that our findings in this article only slightly improve the previously proposed flux density control based on a fractional-order flux density estimator, for which we present a first proof of concept in the near future. However, for the commonly omitted and supposedly minor perturbations like hysteresis, saturation, fringing and leakage fluxes, we provided an extensive review about modeling approaches and discussed their impact as well as practical applicability as reference for future studies.

\section{ACKNOWLEDGMENT}

This work was supported by the German Research Foundation (DFG) under Grant HO 1483/78-1.

\section{REFERENCES}

[1] R. Seifert, K. Röbenack, and W. Hofmann, "Rational approximation of the analytical model of nonlaminated cylindrical magnetic actuators for flux estimation and control," IEEE Transactions on Magnetics, vol. 55, no. 12, pp. 1-16, Dec. 2019.

[2] Z. Whitlow, R. Fittro, and C. Knospe, "Segmented magnetic thrust bearings: Analytic models and predictions of significant improvement in dynamic performance," in 14th International Symposium on Magnetic Bearings, Aug. 2014.

[3] R. Seifert, E. Fleischer, and W. Hofmann, "Experimentelle Bestimmung der Rotorverluste eines dreipoligen kombinierten Radial/Axialmagnetlagers aus Pulververbundwerkstoffen [Experimental determination of the rotor losses of a three-pole combined radial/axial magnetic bearing made of soft magnetic composites]," in 11. Workshop Magnetlagertechnik Zittau-Chemnitz, vol. 11, Sep. 2017, pp. 27-34, (in German).

[4] L. Zhu and C. Knospe, "Modeling of nonlaminated electromagnetic suspension systems," IEEE Transactions on Mechatronics, vol. 15, no. 1, pp. 59-69, Feb. 2010

[5] K. Nonami, W. He, and H. Nishimura, "Robust control of magnetic levitation systems by means of $\mathrm{H}^{\infty}$ control/ $\mu$-synthesis," International Journal Series C, vol. 37, no. 3, pp. 513-520, Sep. 1994.

[6] T. Schuhmann, W. Hofmann, and R. Werner, "Improving operational performance of active magnetic bearings using kalman filter and state feedback control," IEEE Transactions on Industrial Electronics, vol. 59, no. 2, pp. 821-829, 22012.

[7] D. Vischer, "Sensorlose und spannungsgesteuerte Magnetlager [Sensorless and voltage controllod magnetic bearings]," Dissertation, ETH Zürich, 1988, (in German).

[8] F. J. Keith, "Implicit flux feedback control for magnetic bearings," Dissertation, 1993.

[9] J. Ferreira, E. Maslen, and R. Fittro, "Transpermeance amplifier applied to magnetic bearings," Actuators, vol. 6, no. 9, pp. 1-20, Feb. 2017.

[10] G. Schweitzer, H. Bleuler, E. H. Maslen, P. Keogh, R. Larsonneur, R. Nordmann, Y. Okada et al., Magnetic Bearings: Theory, Design, and Application to Rotating Machinery. Springer, 2009.

[11] R. Stoll, The analysis of eddy currents, ser. Monographs in electrical and electronic engineering. Clarendon Press, 1974.

[12] J. J. Feeley, "A simple dynamic model for eddy currents in a magnetic actuator," IEEE Transactions on Magnetics, vol. 32, no. 2, pp. 453-458, Mar. 1996.

[13] R. Rabinovici and B. Kaplan, "Effective magnetization and forces due to eddy currents," IEEE Transactions on Magnetics, vol. 28, no. 3, pp. 1863-1869, May 1992.

[14] L. Kucera and M. Ahrens, "A model for axial magnetic bearings including eddy currents," in 3rd International Symposium on Magnetic Suspension Technology. NASA, Jul. 1996, pp. 421-437.

[15] R. Seifert, C. Steiert, W. Hofmann, and K. Röbenack, "Einführung in die fraktionale Flussschätzung in elektromagnetischen Aktoren [Introduction into fraktional order flux estimation in electromagnetic actuators]," Automatisierungstechnik, vol. 67, p. 572-586, Jul. 2019, (in German).

[16] F. Preisach, "Über die magnetische Nachwirkung [About the magnetic aftereffect]," Zeitschrift für Physik, vol. 94, no. 5, pp. 277-302, 51935 , (in German).

[17] M. Krasnosel'skii, M. Niezgodka, and A. Pokrovskii, Systems with Hysteresis. Springer, 1983. 
[18] I. D. Mayergoyz, "Hysteresis models from the mathematical and control theory points of view," Journal of Applied Physics, vol. 57, no. 8, pp. 3803-3805, Apr. 1985.

[19] G. Bertotti, "Dynamic generalization of the scalar preisach model of hysteresis," IEEE Transactions on Magnetics, vol. 28, no. 5, pp. 2599 2601, Sep. 1992.

[20] E. Della Torre and F. Vajda, "Parameter identification of the completemoving-hysteresis model using major loop data," IEEE Transactions on Magnetics, vol. 30, no. 6, pp. 4987-5000, 111994.

[21] E. Cardelli, E. D. Torre, and B. Tellini, "Direct and inverse preisach modeling of soft materials," IEEE Transactions on Magnetics, vol. 36, no. 4, pp. 1267-1271, Jun. 2000.

[22] E. Cardelli and E. Della Torre, "Modeling of hysteresis and dynamic losses in soft ferrites up to radiofrequency level," Physica B: Condensed Matter, vol. 306, no. 1-4, pp. $240-245$, Sep. 2001.

[23] M. Novak, J. Eichler, and M. Kosek, "Difficulty in identification of preisach hysteresis model weighting function using first order reversal curves method in soft magnetic materials," Applied Mathematics and Computation, vol. 319, pp. 469-485, 22018.

[24] D. C. Jiles, "Modelling the effects of eddy current losses on frequency dependent hysteresis in electrically conducting media," IEEE Transactions on Magnetics, vol. 30, no. 6, pp. 4326-4328, 111994.

[25] E. Cardelli, E. D. Torre, E. Pinzaglia, and A. Massinelli, "Analysis of magnetic losses of cylindrical cores in the frequency domain," IEEE Transactions on Magnetics, vol. 39, no. 3, pp. 1365-1368, May 2003.

[26] D. Ribbenfjard and G. Engdahl, "Novel method for modelling of dynamic hysteresis," IEEE Transactions on Magnetics, vol. 44, no. 6, pp. 854-857, Jun. 2008.

[27] A. M. Milovanovic and B. M. Koprivica, "Mathematical model of major hysteresis loop and transient magnetizations," Electromagnetics, vol. 35, no. 3, pp. 155-166, Mar. 2015.

[28] M. C. Rodriguez and C. Sanz, "Simple frequency domain model for hysteresis and eddy currents in cylindrical and parallelepipedal cores," IEEE Transactions on Magnetics, vol. 43, no. 5, pp. 1912-1919, May 2007.

[29] D. C. Jiles and D. L. Atherton, "Theory of ferromagnetic hysteresis," Journal of Magnetism and Magnetic Materials, vol. 61, no. 1, pp. 48 60, 31986.

[30] G. Bertotti, Hysteresis in magnetism: for physicists, materials scientists, and engineers. Academic press, 1998.

[31] R. Herzog, S. Vullioud, R. Amstad, G. Galdo, P. Muellhaupt, and R. Longchamp, "Self-sensing of non-laminated axial magnetic bearings: Modelling and validation," Journal of System Design and Dynamics, vol. 3, no. 4, pp. 443-452, Jul. 2009.

[32] J. Faiz and S. Saffari, "A new technique for modeling hysteresis phenomenon in soft magnetic materials," Electromagnetics, vol. 30, no. 4, pp. 376-401, May 2010.

[33] C. Monje, Y. Chen, B. Vinagre, D. Xue, and V. Feliu-Batlle, Fractionalorder Systems and Controls: Fundamentals and Applications, ser. Advances in Industrial Control. Springer, 2010.

[34] D. C. Meeker, E. H. Maslen, and M. D. Noh, "An augmented circuit model for magnetic bearings including eddy currents, fringing, and leakage," IEEE Transactions on Magnetics, vol. 32, no. 4, pp. 3219-3227, Jul 1996.

[35] Y. Sun, Y.-S. Ho, and L. Yu, "Dynamic stiffnesses of active magnetic thrust bearing including eddy-current effects," IEEE Transactions on Magnetics, vol. 45, no. 1, pp. 139-149, Jan. 2009.

[36] K. Wang, D. Wang, H. Lin, Y. Shen, X. Zhang, and H. Yang, "Analytical modeling of permanent magnet biased axial magnetic bearing with multiple air gaps," IEEE Transactions on Magnetics, vol. 50, no. 11, pp. $1-4,2014$.

[37] Y. Le and K. Wang, "Design and optimization method of magnetic bearing for high-speed motor considering eddy current effects," IEEE/ASME Transactions on Mechatronics, vol. 21, no. 4, pp. 2061-2072, Aug. 2016.

[38] Y. Zhong, L. Wu, X. Huang, Y. Fang, and J. Zhang, "An improved magnetic circuit model of a 3-dof magnetic bearing considering leakage and cross-coupling effects," IEEE Transactions on Magnetics, vol. 53, no. 11, pp. 1-6, 112017.

[39] H. C. Roters, Electromagnetic Devices. Wiley, 1941. 